\title{
Opportunität und Konsens: Verfahrensförmige Normsuspendierung als Hilfe für die Überlast im Kriminaljustizsystem?
}

\section{A. Die Phänomene Konsensprinzip und Opportunitätsprinzip im Lichte der Sta- tistik}

B. Das Opportunitätsprinzip im deutschen Strafrecht

I. Das Legalitätsprinzip

II. Das Opportunitätsprinzip

III. Die wichtigsten Opportunitätsvorschriften nach herrschender Sicht

1. $\S 153 \mathrm{StPO}$

2. $\S 153$ a StPO

IV. Aufstieg des Opportunitätsprinzips

1. Geschichtlicher Hintergrund

2. Gründe für den Aufstieg des Opportunitätsprinzips

C. Das Konsensprinzip im deutschen Strafrecht

I. Das Konsensprinzip

II. Die wichtigsten konsensualen Elemente in der StPO

1. $\S 153$ a StPO in Abgrenzung zu $\S 153$ Abs. 2 StPO

2. Das Strafbefehlsverfahren, $\S \S 407 \mathrm{ff}$. StPO

a) Kritik am Strafbefehlsverfahren

b) Das Strafbefehlsverfahren als konsensuales Verfahren?

III. Aufstieg des Konsensprinzips am Beispiel des Strafbefehlsverfahrens

1. Geschichtlicher Hintergrund

2. Gründe für den Aufstieg des Konsensprinzips

IV. Absprachenpraxis und gesetzliche Regelung der Verständigung im deutschen Strafverfahren

1. Die Absprache und ihr Weg zu einer gesetzlichen Regelung

2. Kritische Würdigung des neuen Gesetzes (insbesondere des $§ 257$ c StPO)

3. Legitimation von Absprachen?

D. Konflikt des Konsens- und des Opportunitätsprinzips mit tragenden Verfassungs- und Strafrechtsprinzipien

I. Schuldprinzip

II. Gewaltenteilung, Art. 20 Abs. 2 GG und Rechtsprechungsmonopol/Richtervorbehalt, Art. 92 GG

III. Unschuldsvermutung, Art. 6 Abs. 2 EMRK und Rechtsstaatsprinzip

IV. Richterliche Unabhängigkeit, Art. 97 GG (§ 1 GVG, § 25 DRiG)

V. Prinzip der materiellen Wahrheit und Amtsermittlungsgrundsatz

VI. Bestimmtheitsgebot, Art. 103 Abs. 2 GG, $§ 1$ StGB und Gleichheitsgrundsatz, Art. 3 Abs. 1 GG 


\section{E. Rechtfertigung durch andere Legitimationen?}

I. Legitimation durch das Opportunitätsprinzip?

II. Konsensprinzip als Legitimationsgrundlage für den Schuldspruch?

\section{F. Lösungsansatz materiell-rechtliche Entkriminalisierung}

\section{G. Ausblick}

I. Ablehnung formeller Entkriminalisierung mittels Opportunität und Konsens

II. „Königsweg“: Materielle Entkriminalisierung und Rückzug des Strafrechts aus einem präventiven Steuerungsanspruch

\section{A. Die Phänomene Konsensprinzip und Opportunitätsprinzip im Lichte der Statistik}

In welche Richtung entwickelt sich das deutsche Strafverfahren? Diese Frage muss man sich angesichts der Entwicklung der letzten gut 30 Jahre, die mit der kürzlich erfolgten Normierung der Absprachen im Jahre 2009 ihren vorläufigen Höhepunkt (oder besser: Tiefpunkt) gefunden hat, stellen. Anlässlich dieser Pläne des Gesetzgebers erschien bereits am 14.11.2006 eine „Todesanzeige“ in der Süddeutschen Zeitung, in der Heribert Prantl das „Dahinscheiden des klassischen deutschen Strafprozesses (...) nach langem Leiden still und fast unbemerkt in seinem 129. Lebensjahr" betrauert. In diesem Artikel verabschiedet Prantl sich von der deutschen Rechtstradition, von dem Prinzip der materiellen Wahrheit, von dem Amtsermittlungsgrundsatz und von dem Legalitätsprinzip. ${ }^{1}$

Überspitzte Formulierungen oder die erschreckende Wahrheit? Dies gilt es hier zu klären. Untersuchungsgegenstand sind zwei Prinzipien, welche auf der einen Seite zu einer erheblichen Beschleunigung im Strafverfahren führen, aber auf der anderen Seite das rechtsstaatliche Strafrecht nachhaltig bedrohen: Die Rede ist vom Opportunitätsprinzip, welches eine Ausnahme zum Legalitätsprinzip darstellt, und vom Konsensprinzip, welches eine Durchbrechung des kontradiktorischen Verfahrens bedeutet. ${ }^{2}$

Die Geschichte der Reformbemühungen zur Strafprozessordnung zeigt, dass immer mehr Mechanismen entwickelt werden, die die aufwändige Hauptverhandlung zu umgehen versuchen. ${ }^{3}$ Zudem ist die Zunahme abstrakter Gefährdungsdelikte, vielfältiger Pflichtnormierungen im Zuge von Unterlassungs- und Fahrlässigkeitsdelikten und ein Trend zu einem vereinfachten strafrechtlichen Zugriff mit Hilfe von Generalklauseln

1 Prantl, Nicken Sie, nicken Sie!, Süddeutsche Zeitung Nr. 262 vom 14.11.2006, S. 4; ähnlich auch: Strate, Ende oder Wende des Strafzumessungsrechts? Zu den Auswirkungen des Gesetzes über die Verständigung im Strafverfahren, NStZ 2010, S. 364, der den Tod des Strafzumessungsrechts alter Schule beklagt; a. A. Müller, Probleme um eine gesetzliche Regelung der Absprachen im Strafverfahren, 2008, S. 395: es bestehe kein Grund, in den „Abgesang deutscher Rechtstradition einzustimmen".

2 So auch: Fornauf, Die Marginalisierung der Unabhängigkeit der Dritten Gewalt im System des Strafrechts, 2010, S. 171.

3 Fornauf(Fn. 2), S. 142; Weigend, Unverzichtbares im Strafverfahrensrecht, ZStW 113 (2001), S. 275 . 
und unbestimmten Tatbestandsmerkmalen zu beobachten. ${ }^{4}$ Beispiel für diese Entwicklung ist der Tatbestand des $\S 266$ StGB, nach dem eine bloße Vermögensgefährdung bereits ausreicht, um eine Strafbarkeit zu begründen. Diese gesetzgeberische materielle „Kriminalisierungswucht" wird - das ist zu zeigen - durch tiefe Eingriffe in die Struktur des formellen Strafverfahrens ausgeglichen. ${ }^{5}$

In diesem Kontext ist erst einmal zu prüfen, wie sich die Anwendung der Opportunitäts- und Konsensvorschriften in den letzten 30 Jahren entwickelt hat, ${ }^{6}$ um zu verstehen, welche „Macht“ Opportunitäts- und Konsensvorschriften mittlerweile haben. Auffällig ist, dass die größte Zunahme der Einstellungen bei $\S 153 \mathrm{StPO}$ zu verzeichnen ist. Diese Norm verursacht den geringsten Verfahrensaufwand, ${ }^{7}$ ein Umstand, der in Zeiten der Effizienz einen großen Anreiz bietet. Die Zahl der Einstellungen nach § 153 a StPO ist dagegen rückläufig. Grund dafür ist wohl, dass bei Einstellungen nach § 153 a StPO ein vergleichsweise hoher Verfahrensaufwand entsteht, ${ }^{8}$ welcher durch die $\S \S 153 \mathrm{ff}$. StPO doch gerade verhindert werden soll. Die unterschiedliche Entwicklung resultiert somit daraus, dass im Gegensatz zu $\S 153$ a StPO bei $\S 153$ StPO jede spätere Nachprüfung der Auflagen entfällt. ${ }^{9}$

Sowohl die Anklagen als auch die Strafbefehlsanträge haben einen kontinuierlichen prozentualen Rückgang zu verzeichnen. ${ }^{10}$ Ungeachtet dessen haben die absoluten Zahlen seit 1981 zugenommen. Daraus lässt sich schließen, dass der Anteil der schriftlich zu erledigenden Verfahren umso höher ausfällt, je mehr absolute Fälle erledigt werden. Dies zeigt den Bedeutungsverlust des Hauptverfahrens auf. ${ }^{11}$ Ebenfalls bedenklich

4 Albrecht/Hassemer/Voß, Rechtsgüterschutz durch Entkriminalisierung, Vorschläge der Hessischen Kommission „Kriminalpolitik“ zur Reform des Strafrechts, 1992, S.102; Fornauf (Fn. 2), S. 140; Hassemer, Grundlinien eines rechtsstaatlichen Strafverfahrens, KritV 1990, S. 274; Taubald, Konsensuale Erledigung von Strafverfahren in Deutschland und Frankreich, 2009, S. 19.

5 Fornauf (Fn. 2), S. 140; ähnlich auch: Hassemer, Sicherheit durch Strafrecht, StV 2006, S. 326; Albrecht, Eine unabhängige Judikative als Gegengewicht zur Erosion europäischer Strafrechtsprinzipien?, KritV 2010, S. 40 nennt auch $\S 261$ StGB als Tatbestand, bei dem es kaum mehr möglich erscheint, das strafbare Verhalten von einem alltäglichen Normalverhalten zu unterscheiden; Salditt, Wechselwirkungen des formellen und materiellen Strafrechts, in: Duttge/Geilen/Meyer-Goßner/Warda (Hrsg.), Gedächtnisschrift für Ellen Schlüchter, 2002, S. 66: ,anhaltende Produktion einer überhitzten Gesetzgebungsmaschine“.

6 Siehe dazu Statistisches Bundesamt, Staatsanwaltschaften, Arbeitsunterlage; Statistisches Bundesamt, Staatsanwaltschaften, Fachserie 10, Reihe 2.6.

7 Albrecht, Kriminologie. Eine Grundlegung zum Strafrecht, 4., neu bearbeitete Auflage, 2010, S. 207; so auch Systematischer Kommentar zur Strafprozessordnung, mit GVG und EMRK, Wolter (Hrsg.), Band III, §§ 137-197 StPO, 4., neu bearbeitete Auflage, 2011 - SK/Weßlau, $\S 153$, Rn. 3 .

8 Albrecht (Fn. 7), S. 208.

9 Albrecht (Fn. 7), S. 209; ähnlich auch Rieß, Zur weiteren Entwicklung der Einstellungen nach $\S 153$ a StPO, ZRP 1985, S. 216.

101969 kam es zu einem drastischen Rückgang der Strafbefehlsanträge, der mit der Entkriminalisierung der Verkehrsdelikte durch das OWiG 1968 zu erklären ist, Rieß, Zur Entwicklung der Geschäftsbelastung in der ordentlichen Gerichtsbarkeit, DRiZ 1982, S. 206.

11 Albrecht (Fn. 7), S. 207; Heinz, Der Strafbefehl in der Rechtswirklichkeit, in: Britz/Jung/Koriath/Müller (Hrsg.), Grundfragen staatlichen Strafens. Festschrift für Heinz Müller-Dietz zum 70. Geburtstag, 2001, S. 287, 299 sieht auch den zunehmenden Gebrauch des beschleunigten Verfahrens als Grund für den Rückgang des Strafbefehls an. 
stimmt der Umstand, dass laut Fischer in $99 \%$ der Fälle der beantragte Strafbefehl durch den Richter erlassen wird, wobei etwa zwei Drittel der Strafbefehle mangels Einspruchs des Beschuldigten rechtskräftig werden. ${ }^{12}$ Richter „winken“ Strafbefehle demnach schlicht „durch“, woraus schon faktisch eine Verschiebung der Funktionen und Aufgaben vom Richter auf den Staatsanwalt resultiert.

Im Jahr 2010 wurden 21,5\% der Verfahren durch Opportunitätsentscheidungen ${ }^{13}$ und 15,8\% der Verfahren durch Konsensverfahren ${ }^{14}$ erledigt. Die Staatsanwaltschaft ist von der klassischen Anklagebehörde zu einer Behörde mit weitem Sanktionsermessen geworden. ${ }^{15}$ Die Steuerungsansprüche an das Strafrecht wachsen stetig, sodass Ausfilterungsprozesse nötig werden. Der Gesetzgeber hat die Kriminalpolitik daraufhin ausgerichtet, auf prozessualer Ebene gegen die Massenkriminalität vorzugehen und die Opportunitäts- und Konsensvorschriften eingeführt bzw. erweitert. Die Praxis wiederum nimmt diese mangels Alternativen bereitwillig an. Dagegen scheint eine materiellrechtliche Entkriminalisierung dem Gesetzgeber wohl undurchführbar. ${ }^{16}$

\section{B. Das Opportunitätsprinzip im deutschen Strafrecht}

Legalität und Opportunität werden als Gegensatzpaar angesehen: Strenge Gesetzmäßigkeit contra staatsanwaltliches Ermessen. Legalität wird dabei allgemein als der vom Gesetz normierte Regelfall ( $\$ 152$ Abs. 2 StPO), Opportunität als Ausnahme vom Regelfall angesehen. ${ }^{17}$

\section{Das Legalitätsprinzip}

Der Legalitätsbegriff ist abgeleitet von lex (lateinisch: „Gesetz“); ${ }^{18}$ er soll eine strenge Bindung aller Staatsorgane an das Gesetz und das Recht gewährleisten und bildet die

12 Karlsruher Kommentar zur Strafprozessordnung mit GVG, EGGVG und EMRK, Hannich (Hrsg.), 5., neu bearbeitete Auflage, München 2008 - KK/Fischer, vor $\S \S 407$ ff., Rn. 1; so auch Böttcher, Der Strafbefehl auf dem Vormarsch?, in: Böttcher/Hueck/Jähnke (Hrsg.), Festschrift für Walter Odersky zum 65. Geburtstag am 17. Juli 1996, 1996, S. 299.

13 Inbegriffen: $\S \S 153,153$ b, 153 c, 154,154 b, 154 c, 154 d, 154 e StPO, $\S 45$ Abs. 1 , Abs. 2 JGG, § 31 a BtMG; Zahlen von Statistisches Bundesamt, Staatsanwaltschaften 2010, Fachserie 10, Reihe 2.6.

$14 \S 153$ a StPO und $\S \S 407$ ff. StPO; Zahlen von: Statistisches Bundesamt, Staatsanwaltschaften 2010, Fachserie 10, 2.6.

15 Albrecht (Fn. 7), S. 207/208, Überblick zum statistischen Verlauf s. S. 206, Schaubild 17 (Erledigungspraxis der Staatsanwaltsschaft in den Jahren 1981-2008).

16 So auch Albrecht (Fn. 7), S. 209.

17 Horstmann, Zur Präzisierung und Kontrolle von Opportunitätseinstellungen, 2002, S. 26; Shin, Anklagepflicht und Opportunitätsprinzip im deutschen und koreanischen Recht, 1984, S. 27; Schulenburg, Legalitäts- und Opportunitätsprinzip im Strafverfahren, JuS 2004, S. 765.

18 Lieberwirth, Latein im Recht, 5. Auflage, 2007, S. 176. 
Grundlage des klassisch-liberalen rechtsstaatlichen Strafrechts. ${ }^{19}$ Das Legalitätsprinzip in den $\S \S 152$ Abs. 2, 160, 170 Abs. 1 StPO bezeichnet den Strafverfolgungszwang der Staatsanwaltschaft. Diese ist nach $\S 152$ Abs. 2 StPO verpflichtet, wegen aller verfolgbaren Straftaten einzuschreiten, sofern zureichende tatsächliche Anhaltspunkte vorliegen, soweit nicht gesetzlich ein anderes bestimmt ist. Das Legalitätsprinzip gilt nur für mit Kriminalstrafe bedrohte Tatbestände. Im Ordnungswidrigkeitenrecht hingegen gilt das Opportunitätsprinzip. ${ }^{20}$

Das Legalitätsprinzip, welches 1877 mit Inkrafttreten der StPO normiert wurde und sich seitdem unverändert in $\S 152$ Abs. 2 StPO befindet, ${ }^{21}$ war ursprünglich als ein Leitprinzip des deutschen Verfahrensrechts gedacht. Die Ermittlungs- und Anklagezuständigkeit wurde auf die Staatsanwaltschaft übertragen und gleichzeitig die Einführung einer subsidiären Privatklagemöglichkeit abgelehnt. Jedoch hat sich in letzter Zeit das Strafverfolgungssystem immer mehr von Zweckmäßigkeits- und Opportunitätserwägungen leiten lassen. ${ }^{22}$ Der Katalog der Opportunität ist im Laufe der Zeit stetig angewachsen. ${ }^{23}$ So wird heute von einer „Krise des Legalitätsprinzips“ ${ }^{\text {“24 }}$ und von einer „Verwässerung des Legalitätsprinzips “ ${ }^{\circ 2}$ gesprochen und davon, dass das Legalitätsprinzip durchlöchert wie ein Schweizer Käse sei. ${ }^{26}$

Traditionelle Ziele des Legalitätsprinzips sind die Gewährleistung der Gerechtigkeit unabhängig von der Person und deren Ansehen sowie eine größtmögliche Durchsetzung des materiellen Strafanspruchs. Es soll sichergestellt werden, dass begangene Straftaten

19 Döhring, Ist das Strafverfahren vom Legalitätsprinzip beherrscht?, 1999, S. 1; Pott, Die Außerkraftsetzung der Legalität durch das Opportunitätsdenken in den Vorschriften der $\S \S 154$, 154 a StPO, Zugleich ein Beitrag zu einer kritischen Strafverfahrensrechtstheorie, 1999, S. $3 / 33$.

20 Kramer, Grundbegriffe des Strafverfahrensrechts, Ermittlung und Verfahren, 7., überarbeitete Auflage, 2009, S. 183, Rn. 176; Karlsruher Kommentar zum OWiG, Senge (Hrsg.), 3., neu bearbeitete Auflage, 2006 - KK zum OWiG, Einl./Bohnert, Rn. 145.

21 Rose, Der Rechtsschutz des Beschuldigten gegen die Einstellung des Strafverfahrens nach den Opportunitätsvorschriften der Strafprozessordnung, 2006, S. 21; Rie $\beta$, Die Zukunft des Legalitätsprinzips, NStZ 1981, S. 3; Bohnert, Die Abschlussentscheidung des Staatsanwalts, 1992, S. 68; Deiters, Legalitätsprinzip und Normgeltung, 2006, S. 7.

22 Albrecht (Fn. 7), S. 212; Jeutter, Sinn und Grenzen des Legalitätsprinzips im Strafverfahren, 1976, S. $1 / 5$.

23 Döhring (Fn. 19), S. 1; ähnlich auch Erb, Legalität und Opportunität. Gegensätzliche Prinzipien bei der Anwendung von Strafrechtsnormen im Spiegel rechtstheoretischer, rechtsstaatlicher und rechtspolitischer Überlegungen, 1999, S. 22; Lorenzen, Legalitätsprinzip und Opportunitätsprinzip. Kritische Betrachtungen aus und für die Strafverfolgungspraxis, in: Ostendorf (Hrsg.), Strafverfolgung und Strafverzicht. Festschrift zum 125jährigen Bestehen der Staatsanwaltschaft Schleswig-Holstein, 1992, S. 543.

$24 \operatorname{Rieß}$ (Fn. 21), S. 2 ff.

25 Tscherwinka, Absprachen im Strafprozess, 1995, S. 126.

26 Baumann, Grabgesang für das Legalitätsprinzip, ZRP 1972, S. 274. 
nicht unbestraft bleiben und dass Ermittlungen nach streng sachlichen Gesichtspunkten erfolgen. ${ }^{27}$

\section{Das Opportunitätsprinzip}

Die Verpflichtung zur Strafverfolgung gilt gemäß § 152 Abs. 2 StPO nur, ,,soweit nicht gesetzlich ein anderes bestimmt ist". In diesem Halbsatz ist das Opportunitätsprinzip zu sehen. Eine gesetzliche Definition des Opportunitätsprinzips gibt es nicht. Sämtliche Zurückdrängung, Einschränkung oder Begrenzung des Legalitätsprinzips trägt jedoch den Namen Opportunität. ${ }^{28}$ Opportunität bedeutet dem Wortsinn nach ,Zweckmäßigkeit“", abgeleitet vom italienischen Wort opportunitá. ${ }^{29}$ Das lateinische Wort opportunum wird übersetzt mit: „,bequem, vorteilhaft geschickt“", aber auch „bloßgestellt““ 30 Opportunität entstammt als Rechtsbegriff dem Verwaltungsrecht und bedeutet dort die „Rechtsmacht von Verwaltungsbehörden, eigenständig über das Angebrachtsein (die Zweckmäßigkeit) einer hoheitlichen Maßnahme im einzelnen Falle zu entscheiden". 31 Das Opportunitätsprinzip im Strafrecht kann als eine Durchbrechung des Anklagezwanges im Bereich der Bagatell- und mittleren Kriminalität gesehen werden. Nach diesem Prinzip kann ein Strafverfahren z. B. trotz bestehenden Tatverdachts eingestellt werden, wenn bestimmte Voraussetzungen gegeben sind (vgl. $§ \S 153 \mathrm{ff}$. StPO). ${ }^{32} \mathrm{Im}$ Geltungsbereich des Opportunitätsprinzips findet eine Entscheidungsfindung nach Ermessen statt. $^{33}$

\section{Die wichtigsten Opportunitätsvorschriften nach herrschender Sicht}

Der Opportunitätsgedanke kann sowohl in den prozessualen Vorschriften der $\S \S 153$ ff., 374 ff., 383 Abs. 2, 413 ff., 440 StPO, in den $\S \S 45,47$ JGG, $\S 31$ a BtMG, $\S 47 \mathrm{OWiG}$ sowie in den materiellen Vorschriften der $\S \S 248 \mathrm{a}, 257$ Abs. 4, 104 a StGB und in sämtlichen Kronzeugenregelungen erblickt werden. ${ }^{34}$

Die StPO kennt vier Gruppen, in denen das Strafverfahren trotz bestehendem Anfangsverdacht oder hinreichendem Tatverdacht eingestellt werden kann:

27 Rose (Fn. 21), S. 21; Hassemer, Legalität und Opportunität im Strafverfahren, in: Ostendorf (Hrsg.), Strafverfolgung und Strafverzicht. Festschrift zum 125jährigen Bestehen der Staatsanwaltschaft Schleswig-Holstein, 1992, S. 529; Deiters (Fn. 21), S. 3; Weigend, Das „Opportunitätsprinzip“" zwischen Einzelfallgerechtigkeit und Systemeffizienz, ZStW 109 (1997), S. 103/104; Albrecht, Die vergessene Freiheit, Strafrechtsprinzipien in der europäischen Sicherheitsdebatte, 2. Auflage, 2006, S. 94.

28 Horstmann (Fn. 17), S. 51; Kausch, Der Staatsanwalt - Ein Richter vor dem Richter? Untersuchungen zu $§ 153$ a StPO, 1980, S. 61.

29 Aus Jeutter (Fn. 22), S. 7; s. auch Hassemer (Fn. 27), S. 537.

30 Aus Bohnert (Fn. 21), S. 135.

31 Aus Horstmann (Fn. 17), S. 52.

32 Albrecht (Fn. 7), S. 212; Rose (Fn. 21), S. 23.

$33 \operatorname{Erb}$ (Fn. 23), S. 265; Sinner, Der Vertragsgedanke im Strafprozessrecht, 1999, S. 116.

34 Horstmann (Fn. 17), S. 54; Kühne, Strafprozessrecht. Eine systematische Darstellung des deutschen und europäischen Strafverfahrensrechts, 8., völlig neu bearbeitete Auflage, 2010, S. 201, Rn. 310; Sinner (Fn. 33), S. 117. 
- der Tatvorwurf ist gering und es besteht kein Strafverfolgungsinteresse ( $§ 153$, 153 b, 153 c, 154, 154 a, 154 b StPO; hierunter fällt auch $\S 31$ BtMG)

- das Strafverfolgungsinteresse kann auf andere Weise befriedigt werden $(\S 153 \mathrm{a}$ StPO)

- dem Strafverfolgungsinteresse stehen vorrangige staatliche Interessen entgegen $(\S \S 153 \mathrm{~d}, 153 \mathrm{e}, 154 \mathrm{c}, 154 \mathrm{~d}, 154 \mathrm{e})$ oder

- der Verletzte kann die Strafverfolgung selbst betreiben ( $\S 376,377$ StPO). ${ }^{35}$

Die weitere Darstellung nimmt aufgrund ihrer hohen praktischen Relevanz insbesondere die $\S \S 153,153$ a StPO in den Blick.

\section{1. $\S 153 \mathrm{StPO}$}

Die Einstellung nach $\S 153$ StPO ist durch $\S 23$ der Verordnung der Reichsregierung vom 4.1.1924 (Emminger Verordnung) eingeführt worden, ${ }^{36}$ wobei als erster Vorläufer des $\S 153$ SPO bereits die „Allgemeine Verfügung vom 4.4.1854“ bezeichnet werden kann. ${ }^{37} \S 153$ StPO ist die in ihrer Grundstruktur ,schon fast als klassisch zu bezeichnende älteste Begrenzung des Legalitätsprinzips im deutschen Strafverfahrensrecht ". ${ }^{38}$ Bei der Einführung hatte man den Wunsch nach Einsparungen auf dem Gebiet der Strafrechtspflege im Blick. Ursprünglich war das Verfahren schwerpunktmäßig auf den Bereich der Übertretungen ausgerichtet. Es hat seine Bedeutung aber auch nach deren Umwandlung in Ordnungswidrigkeiten beibehalten, indem es weiterhin bei Vergehen von geringer Schwere eine justizentlastende und entkriminalisierende Funktion erfüllt. ${ }^{39} \S 153$ Abs. 1 StPO macht deutlich, dass die Staatsanwaltschaft bei Vorliegen der Voraussetzungen einstellen kann, es aber nicht muss. Hieran wird deutlich, dass es sich um eine Opportunitätsentscheidung handelt.

Zudem verfolgt $§ 153$ StPO den Zweck, eine Ausuferung des materiellen Rechts auf prozessualer Ebene auszugleichen und dient somit einer Entkriminalisierung im Bagatellbereich. ${ }^{40}$ Inzwischen erfasst $\S 153$ StPO die Fälle des eigentlich nicht mehr Strafwürdigen, die aufgrund der Unzulänglichkeiten des materiellen Rechts aber nicht von vornherein ausgeschieden sind. ${ }^{41}$ Der Gesetzgeber verlagert das Problem der Entkriminalisierung so zu sehr in das Prozessrecht. Daran, dass das materielle Strafrecht un-

35 Roxin/Schünemann, Strafverfahrensrecht, Ein Studienbuch, 26., völlig neu bearbeitete Auflage, 2009, S. 71-76, Rn. 5-21.

36 RGBl. 1924 I, S. 15.

37 Aus Männlein, Empirische und kriminalpolitische Aspekte zur Anwendung der Opportunitätsvorschriften $\S \S 153,153$ a StPO durch die Staatsanwaltschaft, 1992, S. 23; Preußisches Justizministerialblatt 1854, S. 147.

38 Sinner (Fn. 33), S. 140; Löwe-Rosenberg, Die Strafprozessordnung und das Gerichtsverfassungsgesetz. Großkommentar, Erb/Esser/Franke/Graalmann-Scheerer/Hilger/Ignor (Hrsg.), 26., neu bearbeitete Auflage, Fünfter Band, $\S \S 151-212$ b, 2008 - LR/Rie $\beta, \S 153$, Rn. 1.

39 LR/Beulke (Fn. 38), § 153, Rn. 1; Heidelberger Kommentar. Strafprozessordnung, Julius u a. (Hrsg.), 4., völlig neu bearbeitete Auflage, 2009 - HK/Gercke, § 153, Rn. 1.

40 Döhring (Fn. 19), S. 239; RGSt 65, 293; LR/Rieß (Fn. 38), § 153, Rn. 1.

41 Kausch (Fn. 28), S. 223. 
verändert bleibt, erkennt man seine Unnachgiebigkeit hinsichtlich der Streichung bestimmter Verhaltensweisen aus dem StGB. ${ }^{42}$

\section{2. $\S 153$ a StPO}

Die Vorschrift des $\S 153$ a StPO wird als „Zentrum des Vertragsdenkens in den Opportunitätsvorschriften der $\S \S 153 \mathrm{ff}$. StPO“ bezeichnet. ${ }^{43}$ Sie wurde durch das EGStGB 1974 eingeführt. Motiv war vor allem die Zurückdrängung des Strafens im Bereich der kleineren Kriminalität, also eine Justizentlastung und eine Entkriminalisierung prozessualer Art. ${ }^{44}$ Jedoch dehnte die Praxis den Anwendungsbereich der Vorschrift im Laufe der Zeit immer mehr auch in den Bereich der mittleren Kriminalität aus. ${ }^{45}$ Im Hinblick auf diese Entwicklung änderte der Gesetzgeber 1993 die Voraussetzung, dass die Schuld gering sein müsse, dahingehend, dass die Schwere der Schuld dem Absehen von der Erhebung der öffentlichen Klage nicht entgegenstehen dürfe. ${ }^{46}$ Wie $\S 153$ StPO eröffnet auch $\S 153$ a StPO einen Ermessensspielraum durch die gesetzliche Aufforderung, bei Vorliegen der gesetzlichen Voraussetzungen den jeweiligen Einzelfall nach dem „öffentlichen Interesse“ zu entscheiden. ${ }^{47}$ Vorstellung des Gesetzgebers bei Einführung der Opportunitätsvorschriften war, dem Bagatellprinzip zu entsprechen. Dies trifft heute jedoch die Wirklichkeit bei weitem nicht mehr, da selbst erhebliche Kriminalität eingestellt wird. Dies belegen siebenstellige Summen, die schon als Leistungen gemäß $\S 153$ a StPO gezahlt werden. ${ }^{48}$

Die Vorschrift stand seit Beginn ihres Inkrafttretens in Kritik, die sich hauptsächlich gegen eine Ausweitung des Opportunitätsprinzips richtete und die pointiert durch das

42 Rzepka, Zur Fairness im deutschen Strafverfahren, 2000, S. 290; ähnlich auch Kudlich, Erfordert das Beschleunigungsgebot eine Umgestaltung des Strafverfahrens? Gutachten C zum 68. Deutschen Juristentag, 2010, C 20.

43 Sinner (Fn. 33), S. 144; ähnlich, aber vorsichtiger LR/Rieß (Fn. 38), § 153 a, Rn. 10, der von „kooperativer Verfahrensbeendigung“" und ,vertragsähnlichen Elementen“ spricht.

44 Vgl. dazu auch BT-Drucks. 7/551, S. 6.

45 Fornauf (Fn. 2), S. 173; Kausch (Fn. 28), S. 17; Fezer, Vereinfachte Verfahren, ZStW 106 (1994), S. 27; LR/Beulke (Fn. 38), § 153, Rn. 3/4; Dahs, § 153 a StPO - ein „Allheilmittel“ der Strafrechtspflege, NJW 1996, S. 1193 spricht in diesem Zusammenhang von dem ,weiten Mantel“" des $\S 153$ a StPO, der überdehnt werde; Saliger, Grenzen der Opportunität und der Fall Kohl. Zugleich Besprechung von LG Bonn, Beschluss vom 28.2.2001, GA 2005, S. 160: „Siegeszug“ des $\S 153$ a StPO.

46 BT-Drucks. 12/1217, S. 19/34; Meyer-Goßner, Beck'sche Kurz-Kommentare. Strafprozessordnung, Gerichtsverfassungsgesetz, Nebengesetze und ergänzende Bestimmungen, 54., neu bearbeitete Auflage, 2011, § 153 a, Rn. 1; Joecks, Strafprozessordnung. Studienkommentar, 3. Auflage, 2011, § 153 a, Rn. 1; Rönnau, Die Absprache im Strafprozess. Eine rechtssystematische Untersuchung der Zulässigkeit von Absprachen nach dem geltenden Strafprozessrecht, 1990, S. 37; Schünemann, Die Verständigung im Strafprozess - Wunderwaffe oder Bankrotterklärung der Verteidigung?, NJW 1989, S. 1896.

$47 \operatorname{Erb}$ (Fn. 23), S. 69.

48 Kühne (Fn. 34), S. 201, Rn. 310; ähnlich Fezer (Fn. 45), S. 27; SK/Weßlau (Fn. 7), § 153 a, Rn. 3, die von Schäden erheblichen Ausmaßes und von politisch bedeutsamen Tatvorwürfen spricht. 
Schlagwort „Freikauf von Strafe“ 49 zusammengefasst werden kann. ${ }^{50}$ Zudem ist auch die Ausübung eines unzulässigen Drucks auf den Beschuldigten unter dem Gesichtspunkt des $\S 136$ a StPO als höchst bedenklich anzusehen. ${ }^{51}$ Weiterhin ist der Anwendungsbereich des $\S 153$ a StPO weitgehend in das Ermessen der Staatsanwaltschaft gestellt, die dadurch einen entscheidenden Einfluss auf die Auslegung der Vergehenstatbestände und auf die Bestimmung der Grenzen des Kriminalstrafrechts bekommt. ${ }^{52}$ Manche gehen sogar so weit, von einer Rückkehr zum Inquisitionsverfahren durch die Vereinigung von Ermittlungs- und Sanktionskompetenz in der Hand des Staatsanwalts zu sprechen. ${ }^{53}$ Kritisch kann man auch sehen, dass bei unklarer Rechtslage eigentlich eine Einstellung nach $\S 170$ Abs. 2 StPO angebracht wäre, da der Beschuldigte möglicherweise unschuldig ist. Stattdessen werden auch solche Verfahren nach $\S 153$ a StPO erledigt und so einem eventuell Unschuldigen eine Sanktion auferlegt. So kann von der Etablierung einer Verdachtsstrafe gesprochen werden. ${ }^{54}$ Auch die Erweiterung durch das RPflEntlG von 1993 ist insofern bedenklich, als dass $\S 153$ a StPO nicht mehr nur auf Bagatellkriminalität anwendbar ist, sondern auch auf Strafsachen, die normalerweise nicht unter die Bagatellkriminalität fallen. ${ }^{55}$

Teilweise wird aufgrund der Auferlegung von Sanktionen in Frage gestellt, ob $\S 153$ a StPO überhaupt eine Opportunitätsvorschrift darstellt. ${ }^{56}$ Dieser Ansicht nach ermächtige das Opportunitätsprinzip nur dazu, Ausnahmen vom Legalitätsprinzip zu machen, d. h., unter bestimmten Voraussetzungen die Strafverfolgung zu unterlassen und auf Sanktionen zu verzichten. Es ermächtige jedoch gerade nicht dazu, an die Stelle der Strafverfolgung andere Sanktionen zu setzen, wie es $\S 153$ a StPO tue. ${ }^{57}$ In diesem Kontext stellt sich die Frage, ob die in $\S 153$ a StPO genannten Auflagen und Weisungen tatsächlich strafrechtlichen Charakter haben oder ob es sich nicht doch um besondere nichtstrafrechtliche Sanktionen handelt. Während einige den Strafcharakter der Auflagen und Weisungen schlicht verneinen, ${ }^{58}$ gibt es andere Stimmen, die der Meinung sind,

49 LR/Beulke (Fn. 38), § 153 a, Rn. 12; Fezer (Fn. 45), S. 26.

50 Kausch (Fn. 28), S. 17; Fezer (Fn. 45), S. 26.

51 Kausch (Fn. 28), S. 40/41; Fezer (Fn. 45), S. 26.

52 Kausch (Fn. 28), S. 42/139 ff.; Fornauf (Fn. 2), S. 174.

53 Rudolphi, Strafprozess im Umbruch. Eine Bilanz der strafverfahrensrechtlichen Reformen seit Kriegsende, ZRP 1976, S. 168; Fezer (Fn. 45), S. 25; Hildebrandt, Gesetzliche Regelung der Verständigung im Strafverfahren. Kann der Strafprozess im Geiste der StPO von 1877 durch den Gesetzgeber gerettet werden?, 2010, S. 136.

54 Hildebrandt (Fn. 53), S. 134; ähnlich Sinner (Fn. 33), S. 144; Salditt, § 153 a StPO und die Unschuldsvermutung, in: Jung/Luxenburger/Wahle (Hrsg.), Festschrift für Egon Müller, 2008, S. 615, der von einem „Freispruchsersatz“ spricht.

55 Fornauf (Fn. 2), S. 173; Böttcher/Mayer, Änderungen des Strafverfahrensrechts durch das Entlastungsgesetz, NStZ 1993, S. 154; Fezer (Fn. 45), S. 31.

56 Kausch (Fn. 28), S. 44; so auch Fornauf (Fn. 2), S. 184.

57 Kausch (Fn. 28), S. 62/65; auch Männlein (Fn. 37), S. 149; Fornauf, Die Unabhängigkeit der Dritten Gewalt im rechtsstaatlichen Strafrecht, KritV 2010, S. 225.

58 Joecks (Fn. 46), § 153 a, Rn. 9; Meyer-Goßner (Fn. 46), § 153 a, Rn. 12; Kremer, Absprachen zwischen Gericht und Verfahrensbeteiligten im Strafprozess, 1994, S. 93; Fezer (Fn. 45), S. 22; SK/Weßlau (Fn. 7), § 153 a, Rn. 8; BGHSt 28, 174, 176. 
dass mit Auflagen und Weisungen durchaus auch Strafzwecke verfolgt werden. ${ }^{59}$ Erstere führen an, dass es sich bei $\S 153$ a StPO um ein Beendigungsverfahren mit Selbstunterwerfung handele und die Auflagen und Weisungen Leistungen seien, die der Genugtuung für das begangene Unrecht dienen. ${ }^{60}$ Die zweite Ansicht argumentiert, dass sich der Strafzweck der Weisungen und Auflagen schon daraus ergebe, dass sie geeignet sein müssen, das öffentliche Interesse an der Strafverfolgung zu beseitigen. Es seien Maßnahmen, mit denen auf Taten reagiert werde, die materielles Unrecht darstellten und diese erfüllten einen Strafzweck. ${ }^{61}$ Fraglich ist jedoch, wie diese Ansicht die Tatsache erklärt, dass die Erfüllung der Auflagen und Weisungen „freiwillig“ erfolgt (die Zustimmung des Beschuldigten ist erforderlich). Der Beschuldigte steht jedoch vor der Wahl, entweder das Strafverfahren durchführen zu lassen mit der Gefahr, eine möglicherweise härtere Strafe zu bekommen oder eben die vergleichsweise leichtere Auflage oder Weisung zu erfüllen. Dadurch wird ein enormer Druck auf den Beschuldigten ausgeübt, sodass die proklamierte Freiwilligkeit wohl kaum mehr als eine Fiktion sein wird. Mit den Auflagen und Weisungen nach $\S 153$ a StPO werden also durchaus auch Strafzwecke verfolgt; sie sind somit strafrechtliche Sanktionen. ${ }^{62}$

Aus diesem Grund ist in $\S 153$ a StPO mangels folgenloser Einstellung keine Opportunitätsvorschrift zu sehen. Vielmehr stellt $\S 153$ a StPO lediglich ein ,unter dem Deckmantel des Opportunitätsprinzips ausgestaltetes flexibles und vermeintlich effektives Verfahren" ${ }^{\prime 63}$ dar. Aufzuzeigen sein wird, dass diese Vorschrift viel eher den Maßgaben eines konsensualen Prozedere folgt.

\section{Aufstieg des Opportunitätsprinzips}

Das Opportunitätsprinzip kann einen stetigen Anwendungszuwachs verzeichnen. Im Folgenden wird der geschichtliche Hintergrund ${ }^{64}$ der Entwicklung der $\$ \S 153$ ff. StPO dargestellt, um darauf aufbauend Gründe und Ursachen für den Aufstieg des Opportunitätsprinzips zu erläutern. Die herrschende Meinung sieht - anders als die Verfasserin - auch $\S 153$ a StPO als Opportunitätsvorschrift. Der Konsensgedanke wird in dieser Vorschrift nur selten gesehen. Im Folgenden wird hinsichtlich der Konjunktur von Opportunität auch die Einstellung unter Auflagen - wie es von der herrschenden Meinung bewertet wird - in die Analyse einbezogen.

59 Kausch (Fn. 28), S. 54; Männlein (Fn. 37), S. 149; Meier, Kriminologie, 4., neu bearbeitete Auflage, 2010, S. 227, Rn. 9; Duttge, Möglichkeiten eines Konsensualprozesses nach deutschem Strafprozessrecht, ZStW 115 (2003), S. 559; Dencker, Die Bagatelldelikte im Entwurf eines EGStGB, JZ 1973, S. 149 f.

60 Meyer-Goßner (Fn. 46), § 153 a, Rn. 12; Kremer (Fn. 58), S. 93; SK/Weßlau (Fn. 38), $\S 153 \mathrm{a}, \mathrm{Rn} .2$.

61 Kausch (Fn. 28), S. 54/55; Rose (Fn. 21), S. 78; Meier (Fn. 59), S. 227, Rn. 9; Fornauf (Fn. 2), S. 185; Sinner (Fn. 33), S. 145.

62 Kausch (Fn. 28), S. 56/57; a. A. SK/Weßlau (Fn. 7), § 153 a, Rn. 9, die den Entscheidungsdruck des Beschuldigten nicht mit der Ausübung von Zwang verwechselt sehen will.

63 Fornauf(Fn. 2), S. 186.

64 Vgl. hierzu umfassend Kausch (Fn. 28). 


\section{Geschichtlicher Hintergrund}

Vor Etablierung eines einheitlichen Strafverfahrensrechts für ganz Deutschland herrschte in den einzelnen landesrechtlichen Strafprozessordnungen ein unscharfes Bild hinsichtlich der Verteilung von Legalität und Opportunität. ${ }^{65}$ Als die 1877 ausgefertigte RStPO 1879 in Kraft trat, hatte sich das Legalitätsprinzip durchgesetzt. ${ }^{66}$ Ausnahmen von diesem waren nur in sehr begrenztem Umfang möglich. ${ }^{67}$ Ein Grund für diese Entwicklung war, dass zum Zeitpunkt des Erlasses der RStPO die Staatsanwaltschaft als Teil der Exekutive dem Monarchen unterstand. Durch die Etablierung des Legalitätsprinzips wollte man gewährleisten, dass die Staatsanwaltschaft jede Straftat ohne Ansehen der Person verfolgen würde. ${ }^{68}$ Jedoch zeigten sich in der Praxis relativ rasch Mängel des starren Legalitätsprinzips. Bereits um die Jahrhundertwende wurde erste deutliche Kritik an der strikten Verfolgungspflicht laut. Im Zuge dessen wurde der in $\S 152$ Abs. 2 StPO genannte Vorbehalt ,soweit nicht gesetzlich ein anderes bestimmt ist" im Laufe der letzten gut hundert Jahre zunehmend durch Einzelvorschriften ausgefüllt. ${ }^{69}$

Seinen entscheidenden Durchbruch hatte das Opportunitätsprinzip jedoch 1924 durch die Emminger Notverordnung, die für das Strafverfahren diverse Durchbrechungen des Verfolgungszwangs vorsah. Nachdem der Opportunitätsgedanke mit den $\S \S 153,154$ RStPO so in das Strafverfahren Eingang gefunden hatte, stand die Tür weit offen für eine zunehmende Zurückdrängung des Legalitätsprinzips. ${ }^{70}$ Das Dritte Reich brachte weitere Einbrüche in das Legalitätsprinzip. So wurde bspw. die Zustimmungspflicht des Richters zur Einstellung nach $\S 153$ RStPO gestrichen und am 13.12.1944 wurde die Staatsanwaltschaft durch eine Verordnung von der Anklagepflicht völlig freigestellt, sodass das Opportunitätsprinzip galt. ${ }^{71}$ Auch nach dem Krieg erfuhr das Legalitätsprinzip andere Einschnitte. Insbesondere die Einführung des $\S 153$ a StPO erweiterte die Einstellungsmöglichkeiten aus Opportunitätsgründen im Bereich kleinerer Kriminalität erheblich. ${ }^{72}$

65 Horstmann (Fn. 17), S. 45; Bohnert (Fn. 21), S. 66.

66 Horstmann (Fn. 17), S. 45/46; Hohendorf, $§ 153$ a StPO als Radikalmittel zur Bewältigung der „Massen-Bagatellkriminalität?““, NJW 1987, S. 1179; Rieß (Fn. 21), S. 3; Schmidt-Jortzig, Möglichkeiten einer Aussetzung des strafverfolgerischen Legalitätsprinzips bei der Polizei, NJW 1989, S. 130.

67 Pott (Fn. 19), S. 7.

68 Roxin/Schünemann (Fn. 35), S. 71, Rn. 2; Shin (Fn. 17), S. 17; Erb (Fn. 23), S. 21; Geppert, Das Legalitätsprinzip, JURA 1982, S. 151.

69 Horstmann (Fn. 17), S. 46/47.

70 Horstmann (Fn. 17), S. 47/48; Hohendorf (Fn. 66), S. 1179; Rieß (Fn. 21), S. 3.

71 Horstmann (Fn. 17), S. 48; Pott (Fn. 19), S. 36; Schmidt-Jortzig (Fn. 66), S. 131.

72 Horstmann (Fn. 17), S. 49/50; Schmidt-Jortzig (Fn. 66), S. 131; Rieß, Entwicklung und Bedeutung der Einstellungen nach § 153 a StPO, ZRP 1983, S. 93. 


\section{Gründe für den Aufstieg des Opportunitätsprinzips}

Eine der wichtigsten Grundlagen des Legalitätsprinzips bildet die absolute Straftheorie (zurückgehend auf die Arbeiten von Kant, Bindung und Hegel) ${ }^{73}$ und das tatbezogene Vergeltungsstrafrecht, welches im 18./19. Jahrhundert vorherrschte. Nach der absoluten Straftheorie im Kant'schen Sinne darf Strafe niemals opportunistischen Zweckerwägungen dienen und der Staat hat zur Herstellung absoluter Gerechtigkeit jede Übertretung des Strafgesetzes ausnahmslos zu bestrafen. Der Täter muss um der Gerechtigkeit willen bestraft werden, das durch ihn verursachte Übel muss ausgeglichen werden und dadurch die gestörte Ordnung wiederhergestellt werden (punitur quia peccatum est = Bestraft wird, weil Unrecht begangen worden ist). ${ }^{74}$ Auch der Reichsgesetzgeber im Jahr 1877 war dieser damals vorherrschenden Auffassung gefolgt, indem er das Legalitätsprinzip lückenlos gewährte. ${ }^{75}$ Jedoch wandelte sich das Strafrecht um die Jahrhundertwende in ein solches primär präventiver Art. Mit dieser Überwindung des Vergeltungsstrafrechts verlor auch der Absolutheitsanspruch teilweise seine Berechtigung. ${ }^{76}$ Den relativen Straftheorien ist gemeinsam, dass die Bestimmung und Ausführung der Strafe nach ihrer Wirkung für die Zukunft erfolgt. Wichtig sind Nützlichkeitserwägungen und Zweckmäßigkeit. ${ }^{77}$ Diese relativen Straftheorien reiben sich jedoch mit einem strengen Legalitätsprinzip, da dieses eine Strafverfolgung in allen und damit auch in den Fällen fordert, in denen eine Strafe weder aus spezial- noch aus generalpräventiver Hinsicht geboten erscheint. ${ }^{78}$

Besonders im Bereich der Kleinkriminalität tritt immer wieder das Argument der Effizienz auf. Die chronische Überlastung von Polizei, Staatsanwaltschaften und Gerichten müsse dazu führen, die begrenzten Kapazitäten für schwere Verstöße zu reservieren. ${ }^{79}$ Vor allem die $\S \S 153,153$ a StPO sollten Möglichkeiten schaffen, die kleinere und mittlere Kriminalität im vereinfachten Verfahren ohne Strafe und Vorbestraftsein bei gleichzeitiger Verfahrensbeschleunigung und Arbeitsentlastung bei Staatsanwälten

73 Kant, Die Metaphysik der Sitten, zweite verbesserte Auflage, 1798-1803 (Nachdruck von 1797); Hegel, Grundlinien der Philosophie des Rechts, 2010 (Nachdruck von 1821); Binding, Die Normen und ihre Übertretungen. Eine Untersuchung über die rechtmäßige Handlung und die Arten des Delikts, 2. Auflage, 1890 (Nachdruck von 1872).

74 Liebs, Lateinische Rechtsregeln und Rechtssprichwörter, 7., vollständig überarbeitete und verbesserte Auflage, 2007, S. 185.

75 Horstmann (Fn. 17), S. 56; Roxin/Schünemann (Fn. 35), S. 71, Rn. 2; Shin (Fn. 17), S. 17; Hassemer (Fn. 27), S. 538.

76 Horstmann (Fn. 17), S. 57; Roxin/Schünemann (Fn. 35), S. 71, Rn. 2; Geppert (Fn. 68), S. 151; Rieß (Fn. 21), S. 4; zurückgehend auf von Liszt, Der Zweckgedanke im Strafrecht, 1948 (Nachdruck von 1882).

77 Horstmann (Fn. 17), S. 42/57; Albrecht (Fn. 7), S. 43; Albrecht, Der Weg in die Sicherheitsgesellschaft. Auf der Suche nach staatskritischen Absolutheitsregeln, 2010, S. 30-33; vertiefend zu den Strafzwecktheorien Campagna, Strafrecht und unbestrafte Straftaten. Philosophische Überlegungen zur strafenden Gerechtigkeit und ihren Grenzen, 2007, S. $73 \mathrm{ff}$.

78 Horstmann (Fn. 17), S. 57/58; Hildebrandt (Fn. 53), S. 69; Geppert, Das Opportunitätsprinzip, JURA 1986, S. 310.

79 Erb (Fn. 23), S. 158/159; Kausch (Fn. 28), S. 16; Männlein (Fn. 37), S. 1/34; Kirch, Das Strafbefehlsverfahren nach dem Strafverfahrensänderungsgesetz 1987. Kritische Anmerkungen, 1987, S. 3. 
und Richtern zu erledigen. ${ }^{80}$ Weiterhin sollen die Opportunitätsvorschriften zu einer Entkriminalisierung auf prozessualer Ebene beitragen und dienen somit auch kriminalpolitischen Zwecken. ${ }^{81}$ Ein weiterer Grund für die Ausweitung des Opportunitätsprinzips ist, dass die auf den schwerwiegenden Durchschnittsfall bezogene Kriminalstrafe in den meisten Fällen der Bagatellkriminalität eine Überreaktion darstellt und nicht im Verhältnis zu der Schwere des begangenen Unrechts steht. Verfahren, die nach $\S \S 153$, 153 a StPO eingestellt werden, werden nicht ins Bundeszentralregister eingetragen. So wird erreicht, dass der Täter nicht für alle Zeiten stigmatisiert wird und dass der Kleinkriminelle besser gestellt wird. ${ }^{82}$ Zudem besteht aufgrund der Masse der Bagatelldelikte durch die Anwendung der Kriminalstrafe die Gefahr, dass das Strafrecht seine abschreckende Wirkung verliert, da beinahe jedermann ein- oder mehrmals bestraft wird und Bestraftsein somit als normal erscheint. ${ }^{83}$ Nach der Streichung von Übertretungen ${ }^{84}$ sowie den Privilegierungen im Bereich der kleinen Eigentums- und Vermögenskriminalität im Jahre 1975 entstand das Bedürfnis nach einem prozessualen Ausgleich. Auch dies gab Anlass zur Neuregelung der $\S \S 153,153$ a StPO, da die Notwendigkeit entstand, eine strengere Beurteilung dieser Form der Bagatellkriminalität zu vermeiden. ${ }^{85}$

\section{Das Konsensprinzip im deutschen Strafrecht}

\section{Das Konsensprinzip}

Im deutschen Strafverfahren zeigt sich immer mehr die Tendenz, Verfahren durch die Übereinkunft der Parteien zu erledigen. Gewisse Formen des Konsenses bzw. des Vertragsgedankens dringen somit in das Strafverfahrensrecht ein. ${ }^{86}$ Konsens, abgeleitet

80 Hohendorf (Fn. 66), S. 1177; Kausch (Fn. 28), S. 16; Entw. EGStGB BT-Drucks. VI/3250, S. 283.

81 Siehe besonders hinsichtlich $\S 153$ a StPO BT-Drucks. 7/550, S. 298 und 7/551, S. 69.

82 Kausch (Fn. 28), S. 21; Männlein (Fn. 37), S. 2/35; Hildebrandt (Fn. 53), S. 132; ähnlich auch LR/Beulke (Fn. 38), § 153, Rn. 1; Bandemer, Einstellung hinter der Einstellung. Absehen von Strafverfolgung nach § 154 I Nr. 1 StPO bei Einstellung nach § 153 a StPO im Bezugsverfahren, NStZ 1988, S. 299.

83 Kausch (Fn. 28), S. 21; ähnlich Weigend (Fn. 27), S. 111.

84 Fezer (Fn. 45), S. 24: Das EGStGB hatte die unterste Deliktskategorie der „Übertretung“ abgeschafft, sodass Bagatellfälle von Eigentums- und Vermögensdelikten zu Vergehen hochgestuft wurden; die Entkriminalisierung sollte nun im Prozessrecht stattfinden.

85 Shin (Fn. 17), S. 38; Kaiser/Meinberg, „Tuschelverfahren“ und „Millionärsschutzparagraph“ - Empirische Erkenntnisse zur Einstellung nach § 153 a I StPO am Beispiel Wirtschaftskriminalität, NStZ 1984, S. 344; Fezer (Fn. 45), S. 24.

86 Sinner (Fn. 33), S. 23, der jedoch auf S. 75 klarstellt, dass Vertrag und Konsens nicht gleichbedeutend seien: Der Vertrag setze einen Rechtsbindungswillen voraus, was der Konsens gerade nicht tue; Landau, Verfahrensabsprachen im Ermittlungsverfahren, DRiZ 1995, S. 134; Kintzi, Verständigungen im Strafverfahren. Überlegungen zu den Thesen und dem Gutachten der Großen Strafrechtskommission, JR 1990, S. 314, Weichbrodt, Das Konsensprinzip strafprozessualer Absprachen, 2006, S. 286. 
vom Lateinischen consensus, bedeutet „(Willens-) Übereinstimmung, Zustimmung ${ }^{\text {“ }} .{ }^{87}$ Konsens kann als ein Legitimationsprinzip verstanden werden. ${ }^{88}$ Er wird auch als Methode betrachtet, die Wahrheitsfindung anders als bisher üblich und zugleich prozessökonomischer zu gestalten als mit dem am Amtsaufklärungsgrundsatz orientierten Verfahren. ${ }^{89}$ Das Konsensprinzip ist somit ein Oberbegriff für all solche Erledigungsformen, bei denen die Prozessbeteiligten, insbesondere der Beschuldigte, entweder einem bestimmten Verfahrensergebnis zustimmen müssen oder aber der Beschuldigte durch seine Zustimmung die Wahl einer besonderen Verfahrensart ermöglicht. ${ }^{90}$

Die Strafprozessordnung enthält eine Reihe konsensualer Elemente. Insbesondere $\S 153$ a StPO, das Strafbefehlsverfahren und die neue Regelung der Verständigung zeigen, dass eine Verständigung zwischen den Verfahrensbeteiligten über das Ergebnis und die Erledigung des Strafverfahrens der Strafprozessordnung nicht völlig fremd ist. ${ }^{91}$ Ziel für die Suche nach einem Konsens ist dabei entweder, die Hauptverhandlung völlig zu vermeiden wie bei $\S 153$ a StPO oder beim Strafbefehl, oder die Hauptverhandlung schneller abzuwickeln wie bei einer Absprache. In allen Fällen soll die Zustimmung des Beschuldigten den Konsens legitimieren. ${ }^{92} \mathrm{Ob}$ dies wirklich der Fall sein kann, wird im Folgenden untersucht.

\section{Die wichtigsten konsensualen Elemente in der StPO}

\section{1. $\S 153$ a StPO in Abgrenzung zu $\S 153$ Abs. 2 StPO}

Gesetzlicher Hauptanknüpfungspunkt für die Vermutung eines konsensorientierten Verfahrens bei $\S 153$ a StPO ist das Tatbestandsmerkmal der „Zustimmung des Beschuldigten". Zudem wird in der Erfüllung der Auflagen und Weisungen eine konkludente Einwilligung gesehen. Erst durch die Bereitschaft des Beschuldigten könne das Verfahren letztlich eingestellt werden. ${ }^{93}$ Sinner sieht eine „Austauschbeziehung“ in der Erfüllung der Auflagen und Weisungen durch den Beschuldigten, „,im Tausch“ gegen das endgültige Absehen von Strafverfolgung bzw. die endgültige Einstellung des Strafverfahrens. ${ }^{94}$ Nach ihm komme der Vertragsgedanke in $\$ 153$ a StPO zweifach zum Ausdruck: Einmal in einem „Verpflichtungsgeschäft“, das durch das Einverständnis der

87 Schorer, Das Konsensprinzip in der internationalen Gerichtsbarkeit, 2003, S. 29; Lieberwirth (Fn. 18), S. 69; etymologisch deutet der Begriff auch auf unerfreuliche Erscheinungen hin wie die einer konspirativen Verschwörung oder eines Komplotts, s. Langenscheidts, Handwörterbuch Lateinisch-Deutsch, 7. Auflage, 1997, Stichwort: ,consensus“.

88 Weßlau, Das Konsensprinzip im Strafverfahren - Leitidee für eine Gesamtreform?, 2002, S. 14; Legitimation soll dann die Zustimmung des Beschuldigten gewähren.

89 Weßlau (Fn. 88), S. 17/30.

90 Weßlau (Fn. 88), S. 31.

91 Saal, Absprachen im deutschen und polnischen Strafprozess. Eine rechtsvergleichende Darstellung des Konsensualverfahrens, 2009, S. 4; Kremer (Fn. 58), S. 91; Ioakimidis, Die Rechtsnatur der Absprache im Strafverfahren, 2001, S. 33; Duttge (Fn. 59), S. 555; BGHSt 43, 195, 203; Der Reg.entw. BT-Drucks. 16/12310, S. 8 bezweifelte dies jedoch immer noch.

92 Fornauf(Fn. 2), S. 142.

93 Fornauf(Fn. 2), S. 170/171; ders. (Fn. 57), S. 225.

94 Sinner (Fn. 33), S. 146. 
Beteiligten begründet werde, nach $\S 153$ a StPO zu verfahren. Dieser Verpflichtung folge das „Erfüllungsgeschäft“, indem die Justizorgane das Verfahren einstellen, nachdem der Beschuldigte die Auflagen und Weisungen erfüllt habe. ${ }^{95}$

Fraglich könnte jedoch sein, ob $\S 153$ StPO unter diesen Gesichtspunkten nicht doch Elemente des Konsenses aufweist. Dies könnte insoweit zu bejahen sein, als die Einstellung auch von der Zustimmung des Angeklagten abhängig ist, §153 Abs. 2 StPO. ${ }^{96}$ Dieses Erfordernis soll sicherstellen, dass er die Durchführung des Verfahrens zum Zwecke einer ihm günstigeren Entscheidung erzwingen kann, auch wenn er dabei das Risiko einer Verurteilung in Kauf nimmt. ${ }^{97}$ Es muss demnach zwischen den Verfahrensbeteiligten ein Konsens über die Verfahrenseinstellung erzielt werden, weil sie nicht gezwungen sind, ihre Zustimmung zum Verfahren nach $§ 153$ Abs. 2 zu erteilen. Der Angeklagte verzichtet auf ein Verfahren, das auch mit einem Freispruch enden könnte. Dafür verzichtet der Staat auf die Durchsetzung des öffentlichen Strafanspruchs. ${ }^{98}$

Das Verfahren nach $\S 153$ Abs. 2 StPO soll zum einen der Prozessökonomie dienen, zum anderen der Entkriminalisierung auf strafprozessualem Wege. ${ }^{99}$ Jedoch ist diese Erledigungsform nicht mit der des $\S 153$ a StPO vergleichbar, da es eben nicht darum geht, eine belastende Rechtsfolge zu legitimieren und zudem - im Gegensatz zu $\S 153$ a StPO - kein öffentliches Interesse an der Strafverfolgung besteht. Vielmehr stellt $\S 153$ StPO schlicht eine Opportunitätsvorschrift dar, ${ }^{100}$ der zwar ein Hauch von Konsens anhaftet, der jedoch nicht das Konsensprinzip zugrunde liegt.

\section{Das Strafbefehlsverfahren, $\S \S 407 \mathrm{ff}$. StPO}

Das Strafbefehlsverfahren ist ein Verfahren zur schnellen Bewältigung leichterer Kriminalität und erlaubt bei Vergehen ein summarisches Verfahren vor dem Amtsgericht. Besonders an diesem Verfahren ist, dass es sich um ein schriftliches Verfahren ohne mündliche und öffentliche Hauptverhandlung handelt. ${ }^{101}$

95 Sinner (Fn. 33), S. 149; ähnlich auch Saliger (Fn. 45), S. 167.

96 Bei $\S 153$ Abs. 1 StPO ist dagegen die Zustimmung des Beschuldigten nicht erforderlich, da das Absehen von der Strafverfolgung ihn nicht beschwert.

97 Sinner (Fn. 33), S. 142/143; Meyer-Goßner (Fn. 46), § 153, Rn. 26.

98 Weßlau (Fn. 88), S. 39; Sinner (Fn. 33), S. 144; Ioakimidis (Fn. 91), S. 34.

99 Sinner (Fn. 33), S. 143/144; HK/Gercke (Fn. 39), § 153, Rn. 1; LR/Rieß (Fn. 38), § 153, Rn. 1.

100 Weßlau (Fn. 88), S. 39; Duttge (Fn. 59), S. 558.

101 Kramer (Fn. 20), S. 314, Rn. 321; Schroeder, Strafprozessrecht, 4., neu bearbeitete Auflage, 2007, S. 128, Rn. 191; Müller, Das Strafbefehlsverfahren ( $\S 407$ ff. StPO), Eine dogmatisch-kriminalpolitische Studie zu dieser Form des schriftlichen Verfahrens unter besonderer Berücksichtigung der geschichtlichen Entwicklung - zugleich ein Beitrag zum StVÄG 1987, 1993, S. 56; Fezer (Fn. 45), S. 21 sieht die Bezeichnung „summarisches Verfahren“ als verfehlt an. 
a) Kritik am Strafbefehlsverfahren

Das Strafbefehlsverfahren sieht sich einer Reihe von Kritikpunkten ausgesetzt. Die Rechtsstellung des Beschuldigten kann verkürzt werden, wenn Strafen vorschnell und ohne rechtliches Gehör festgesetzt werden, der Beschuldigte sich aber aus verschiedenen Gründen nicht dagegen wehrt. ${ }^{102}$ Zudem ist eine Beeinträchtigung der Strafrechtspflege möglich, wenn eine Strafe möglichst niedrig bemessen wird, um den Beschuldigten von einem Einspruch abzuhalten. ${ }^{103}$ Demgegenüber riskiert der Angeklagte, der den Strafbefehl nicht akzeptiert, dass die Strafe nach einer Hauptverhandlung höher ausfällt als vom Strafbefehl vorgesehen. ${ }^{104}$

Außerdem kann das Prinzip der Unmittelbarkeit mit Zustimmung des Angeklagten, des Verteidigers und des Staatsanwalts außer Kraft gesetzt werden, $\S \S 411$ Abs. 2 S. 2, 420 Abs. 1, 2 StPO, sodass das Beweisantragsrecht praktisch abgeschafft wird. Somit erkauft sich der Angeklagte das beschleunigte Verfahren durch eine Herabsetzung der Prüfungsvoraussetzungen. ${ }^{105}$ Durch $\S 408$ a StPO wird der Unmittelbarkeitsgrundsatz über $\S \S 251$ ff. StPO hinaus weiter eingeschränkt. ${ }^{106}$ Auch liegen Verstöße gegen das Mündlichkeits- und das Öffentlichkeitsprinzip vor.

\section{b) Das Strafbefehlsverfahren als konsensuales Verfahren?}

Fraglich ist, ob es sich beim Strafbefehlsverfahren tatsächlich um ein konsensuales Verfahren handelt. In diesem Kontext ist zunächst zu klären, ob für den Erlass eines Strafbefehls ein hinreichender Tatverdacht ausreichend ist oder ob der Richter von der Schuld des Täters überzeugt sein muss. ${ }^{107}$ Das ist umstritten. Nach einer Ansicht ist auch für den Strafbefehl der positive Nachweis der Schuld auf der Grundlage der materiellen Wahrheit erforderlich. ${ }^{108}$ Andere sehen das rechtsstaatliche Defizit des Strafbefehlsverfahrens gerade auch in dem Verzicht auf volle Schuldüberzeugung des Richters. Dieser Meinung nach ist bereits ausreichend, dass der Beschuldigte hinreichend tatverdächtig ist. Nach dieser zu bevorzugenden Ansicht kommt es somit zu einer Strafe, ohne dass die Schuld des Angeklagten positiv nachgewiesen wird, sodass eine auf Ver-

102 Roxin/Schünemann (Fn. 35), S. 489, Rn. 2; Löwe-Rosenberg, Die Strafprozessordnung und das Gerichtsverfassungsgesetz. Großkommentar, Erb/Esser/Franke/Graalmann-Scheerer/ Hilger/Ignor (Hrsg.), 26., neu bearbeitete Auflage, Achter Band, §§ 374-448, 2009 - LR/ Gössel, Vor $\S 407$, Rn. 26.

103 Roxin/Schünemann (Fn. 35), S. 489, Rn. 2.

104 Volk, Grundkurs StPO, 7., überarbeitete Auflage, 2010, S. 295, Rn 1.

105 Meyer-Goßner (Fn. 46), vor $\S 407$, Rn. 1; es liegt ein Verstoß gegen $\S 250$ StPO vor.

106 Ambos, Verfahrensverkürzungen zwischen Prozessökonomie und „fair trial“. Eine Untersuchung zum Strafbefehlsverfahren und zum beschleunigten Verfahren, JURA 1998, S. 289.

107 Fornauf(Fn. 2), S. 151; Weßlau (Fn. 88), S. 53; ausführlich dazu s. Geis, Überzeugung beim Strafbefehlserlass?, 2000; die Klärung dieser Frage ist insoweit relevant, als dass nach der ersten Auffassung kein Bedarf für das Konsensprinzip entstehen würde, da der Schuldspruch durch das Schuldprinzip legitimiert würde.

108 So vertreten von Müller (Fn. 101), S. 132 ff.; KK/Fischer (Fn. 12), § 408, Rn. 15; LR/Gössel (Fn. 102), Vor § 407, Rn. 25; Ambos (Fn. 106), S. 288; Fezer (Fn. 45), S. 17/20. 
dacht beruhende Rechtsfolge verhängt wird. ${ }^{109}$ In der nachträglichen Zustimmung des Angeklagten wird dann ein Konsens erblickt, der den fehlenden Schuldnachweis kompensieren soll. ${ }^{110}$ Die Legitimation soll also auf dem Konsens zwischen Staat und Angeklagtem gründen. ${ }^{11}$

Die Konsensorientierung im Strafbefehlsverfahren zeigt sich somit darin, dass der Angeklagte durch den stillschweigenden Verzicht auf einen Einspruch in die gegen ihn verhängte Strafe einwilligt, sodass sein persönliches Interesse und das prozessökonomische Interesse der Justiz sich decken. ${ }^{112}$ Staatsanwalt, Richter und Angeklagter müssen sich einig sein, dass die im Strafbefehl bezeichnete Tat rechtlich richtig qualifiziert und die darin festgesetzte Strafe angemessen ist. ${ }^{113}$ Das Strafbefehlsverfahren stellt somit ein konsensuales Verfahren dar.

Das Konsenselement ist der StPO im Übrigen auch nicht fremd. Andere konsensuale Vorschriften der StPO sind: ${ }^{114} \S \S 380$ Abs. 1, 391 StPO, ${ }^{115} \S 153$ b Abs. 2 StPO, $\S 153$ e StPO, $\S 495$ StPO, $\S 303$ StPO und $\S 420$ Abs. 3 StPO. ${ }^{116}$

\section{Aufstieg des Konsensprinzips am Beispiel des Strafbefehlsverfahrens}

\section{Geschichtlicher Hintergrund}

Das Strafbefehlsverfahren kann auf eine lange Entwicklung zurückblicken. Als erste gesetzliche Regelung des Strafbefehlsverfahren wird $\S 122$ des preußischen Gesetzes vom 17.6.1846 angesehen (damals noch: „Mandatsverfahren“). 1877 wurde das Strafbefehlsverfahren schließlich in die RStPO aufgenommen. Der Anwendungsbereich war damals auf Übertretungen und minder schwere Vergehen beschränkt. ${ }^{117}$ In den Jahren

109 Fornauf(Fn. 2), S. 156 f.; Meyer-Goßner (Fn. 46), Vor § 407, Rn. 1; Schünemann, Absprachen im Strafverfahren? Grundlagen, Gegenstände und Grenzen, Gutachten B für den 58. Deutschen Juristentag, 1990, B $69 \mathrm{f}$.

110 Fornauf (Fn. 2), S. 157/158; ob dies dann jedoch tatsächlich der Fall ist, wird an späterer Stelle geklärt; andere sehen in der Hinnahme des Strafbefehls durch den Beschuldigten auch ein nachträgliches Geständnis und wollen so das Strafbefehlsverfahren noch über die materielle Wahrheit retten. Dies ist jedoch abzulehnen, näheres dazu s. Weßlau (Fn. 88), S. 55.

111 Fornauf(Fn. 57), S. 224; Fornauf (Fn. 2), S. 159.

112 Sinner (Fn. 33), S. 165/166; Kirch (Rn. 79), S. 6; ähnlich auch Bode, Verständigung im Strafprozess, DRiZ 1988, S. 283.

113 Bode (Fn. 112), DRiZ 1988, S. 283.

114 Die Vorschriften werden hier der Vollständigkeit halber genannt. Aus Platzgründen muss vorliegend eine nähere Darstellung der einzelnen Vorschriften unterbleiben.

115 Wobei bei diesem, wie Duttge (Fn. 59), S. 555 richtig erkennt, gerade kein „öffentliches Interesse" an der Strafverfolgung vorliegt, arg. aus $\S 376$ StPO.

116 Aus Sinner (Fn. 33), S. 150-155, S. 159-163; Tscherwinka (Fn. 25), S. 51-63; Meyer-Goßner (Fn. 46), Einl., Rn. 119 a; Müller (Fn. 1), S. 21-29; Duttge (Fn. 59), S. 554 ff. und S. 556, Rn. 101 mit weiteren Nachweisen. Duttge weist jedoch auch hier darauf hin, dass diesen Zustimmungs- und Verzichtserklärungen keinerlei Ersetzung des Wahrheits- durch ein Konsensprinzip zugrunde liege, sondern sie durch die fehlende Relevanz der Aufklärungspflicht gekennzeichnet seien.

117 Müller (Fn. 101), S. 168; Kirch (Rn. 79), S. 3/4; Ebert, Der Tatverdacht im Strafverfahren unter spezieller Berücksichtigung des Tatnachweises im Strafbefehlsverfahren, 2000, S. 200; Heinz (Fn. 11), S. 274. 
1915 und 1917 sowie während des ersten Weltkrieges wurde der Anwendungsbereich des Strafbefehlsverfahrens jeweils erweitert. Auch die wirtschaftliche Not der Nachkriegszeit und die dadurch bedingt anwachsende Kriminalität machten eine Entlastung der Strafjustiz notwendig. Vor allem die Emminger Verordnung 1924 führte zu einer beträchtlichen Ausdehnung des Strafbefehlsverfahrens auf den Bereich der mittleren Kriminalität und das Strafbefehlsverfahren wurde in den $\S \S 407$ ff. StPO geregelt. ${ }^{118}$ Auch das Dritte Reich und die Kriegsgesetzgebung des Zweiten Weltkrieges führten zu einer erneuten Ausdehnung des Anwendungsbereichs; so wurden sogar Verbrechen mit dem Strafbefehlsverfahren erledigt. Das Strafbefehlsverfahren nahm jetzt praktisch die beherrschende Rolle in der Strafrechtspflege ein. ${ }^{119}$ Durch das EGStGB von 1974 wurde der Anwendungsbereich trotz Wegfalls der primären Freiheitsstrafe in anderer Weise erweitert, ebenso durch das StVÄG 1979. ${ }^{120}$ Auch das StVÄG von 1987 und das RPflEntlG 1993 brachten viele Änderungen und Erweiterungen des Anwendungsbereichs. ${ }^{121}$

Zusammenfassend ist festzuhalten, dass der Anwendungsbereich des Strafbefehlsverfahrens seit seiner Einführung mit fast jeder Reform erweitert wurde und von der kleineren Kriminalität bis weit in den Bereich mittlerer Kriminalität ausgeweitet wurde. ${ }^{122}$ Das Strafbefehlsverfahren wird mittlerweile so oft angewendet, dass ein erheblicher Teil der Fälle die einfache und klare Beweislage, die grundsätzlich erforderlich ist, sicherlich nicht aufweist. ${ }^{123}$

\section{Gründe für den Aufstieg des Konsensprinzips}

Wesentlicher Grund für das Auftauchen der konsensualen Elemente stellt die Ausweitung des materiellen Strafrechts - infolge der Einsetzung des Strafrechts als praktisches Steuerungsinstrument - und die daraus resultierende Überlastung des Kriminaljustizsystems dar. Ebenso wie die Opportunitätsvorschriften stellen Konsensvorschriften Erledigungsstrategien dar, die auf Effizienz und Flexibilität angelegt sind. ${ }^{124}$ Sie werden notwendig, da die Justiz nicht in der Lage ist, alle anfallenden Straftaten in einer Hauptverhandlung mit vollständiger Beweisaufnahme zu erledigen. ${ }^{125}$ Mit Hilfe konsensualer Verfahren sollten und sollen einfach gelagerte Fälle von geringer Tatschwere schneller erledigt werden. ${ }^{126}$

118 Müller (Fn. 101), S. 242-244; Ebert (Fn. 117), S. 201; Vivell, Das Strafbefehlsverfahren nach Eröffnung des Hauptverfahrens ( $(408$ a StPO), 2006, S. 34.

$119 \operatorname{Müller}$ (Fn. 101), S. 247/248; Ebert (Fn. 117), S. 201; bereits 1950 wurde jedoch die Strafbefehlsfähigkeit von Verbrechen wieder beseitigt, Kirch (Fn. 79), S. 21.

120 Müller (Fn. 101), S. 252-254.

121 Vgl. auch Rieß, Zweifelsfragen zum neuen Strafbefehlsverfahren, JR 1988, S. 133; Vivell (Fn. 118), S. 42; KK/Fischer (Fn. 12), Vorb. zu § 407, Rn. 2.

122 Vivell (Fn. 118), S. 40-44; Fornauf (Fn. 2), S. 149; Heinz (Fn. 11), S. 273.

123 Weßlau, Absprachen im Strafverfahren, ZStW 116 (2004), S. 159.

124 Fornauf(Fn. 57), S. 224; Sinner (Fn. 33), S. 118; Weigend, Abgesprochene Gerechtigkeit Effizienz durch Kooperation im Strafverfahren?, JZ 1990, S. 780; Kirch (Fn. 79), S. 3, zusätzlich wurde auch noch das beschleunigte Verfahren in die RStPO aufgenommen.

125 Weßlau (Fn. 88), S. 65; Duttge (Fn. 59), S. 560.

126 KK/Fischer (Fn. 12), Vorb. zu § 407, Rn. 1. 
Der Wandel der Strafzwecke wird auch hier als Grund für den Aufstieg der Konsensvorschriften genannt. Wiedergutmachung und Resozialisierung seien nur im Wege des Konsenses sinnvoll umzusetzen, während die Strafzwecke der Vergeltung und Sühne sich der Realisierung durch den Konsens entzögen. ${ }^{127}$ Die Entwicklung zu mehr Konsensorientierung wird auch als mit dem Wesen pluralistischer Demokratievorstellungen untrennbar verbunden angesehen. Erst in der Demokratie werde die Zustimmung der Mehrheit zu einem notwendigen Ausgangspunkt für die Entscheidung und das Handeln der Gesamtheit. ${ }^{128}$ Sowohl das Verfahren nach $\S 153$ a StPO als auch das Strafbefehlsverfahren sollen zudem den Interessen des Beschuldigten gerecht werden. Bei ersterem geht es darum, dass ein Strafverfahren ohne „wirkliche Strafe“ erledigt wird und der Beschuldigte nicht als vorbestraft gilt. Bei letzterem geht es um die Vermeidung der Belastungen einer öffentlichen Hauptverhandlung. ${ }^{129}$ Teilweise wird in diesem Kontext auch eine Orientierung an Lösungsstrategien anderer Rechtsordnungen („,common law") genannt. ${ }^{130}$

\section{Absprachenpraxis und gesetzliche Regelung der Verständigung im deutschen Strafverfahren ${ }^{131}$}

1. Die Absprache und ihr Weg zu einer gesetzlichen Regelung

Mit dem Begriff Absprache ${ }^{132}$ können viele Formen einer Verständigung zwischen den Verfahrensbeteiligten gemeint sein. Verständigungen sind während des Ermittlungsverfahrens oder während des Hauptverfahrens möglich. Ziel einer Absprache ist jedoch immer, eventuell bestehende Reibungspunkte zwischen den Strafverfolgungsbehörden und dem Beschuldigten zu beseitigen und dadurch die Abwicklung des Verfahrens zu beschleunigen. ${ }^{133}$ Dies wird ermöglicht, indem seitens des Angeklagten ein Geständnis und seitens des Verteidigers die Rücknahme oder das Nichtstellen eines Beweisantrags in Aussicht gestellt wird, um dafür als „Gegenleistung“ mit der Staatsanwaltschaft oder

127 Lüderssen, Die Verständigung im Strafprozess. Überlebensstrategie oder Paradigmawechsel?, StV 1990, S. 417; ähnlich für Absprachen: Schünemann (Fn. 46), S. 1898.

128 Jahn, Zurück in die Zukunft - Die Diskurstheorie des Rechts als Paradigma des neuen konsensualen Strafverfahrens, GA 2004, S. 276, der darin den Beginn der „Karriere“ des Konsensbegriffs in der deutschen Staatsrechtslehre sieht und die Diskurstheorie des Rechts als Paradigma des neuen konsensualen Strafverfahrens ansieht; Müller (Fn. 1), S. 395.

129 Fornauf (Fn. 2), S. 171; KK/Schoreit (Fn. 12), § 153 a, Rn. 1; Weßlau (Fn. 123), S. 150.

130 Hanack, Vereinbarungen im Strafprozess. Ein besseres Mittel zur Bewältigung von Großverfahren?, StV 1987, S. 504; Vogler, Konsensuale Elemente im Strafprozess in England und Wales sowie in den USA, ZStW 116 (2004), S. 136.

131 BGBl. I 2009, S. 2353 f.

132 Im Folgenden wird der Begriff „Absprache“ verwendet; im Rahmen der gesetzlichen Neuregelung wird der Begriff „Verständigung“" verwendet; zu weiteren Begriffen Tschwernika (Fn. 25), S. 20.

133 Hauer, Geständnis und Absprache, Berlin 2007, S. 47; in diesem Punkt geht es nur um Absprachen im Hauptverfahren. 
dem Gericht über die Höhe des Strafmaßes, eine Strafaussetzung zur Bewährung etc. zu verhandeln. ${ }^{134}$

In der Vergangenheit waren Absprachen im Strafverfahren nicht gesetzlich geregelt, sie existierten jedoch praeter legem bzw. contra legem, ${ }^{135}$ es galt weitestgehend Richterrecht. Das erste Auftauchen der Absprachenpraxis wird für Mitte/Ende der siebziger Jahre angenommen. Mit dem 58. Deutschen Juristentag 1990 etablierten sich die Absprachen im Strafverfahren dann endgültig als ein zentraler Gegenstand der deutschen strafprozessualen Diskussion. ${ }^{136}$ Durch die immer höhere Anzahl von Strafsachen war der Druck auf die Strafjustiz groß geworden. Der Gesetzgeber blieb jedoch passiv, sodass die Rechtsprechung sich selber half, indem sie Verständigungen zwischen Gericht und Verfahrensbeteiligten zuließ. ${ }^{137}$ Daraufhin konnte sich die Absprachenpraxis immer weiter ausbreiten und nahm ungeahnte - weil unüberprüfbare - Ausmaße an. Weigend sprach bereits vor gut 20 Jahren davon, dass das Abspracheverfahren in manchen Bereichen das herkömmliche „streitige“ Verfahren weitgehend verdrängt habe. ${ }^{138}$ Deutsche Gerichte seien von der „Droge“ Absprache inzwischen so süchtig, dass mit einer Entwöhnung nicht zu rechnen sei. ${ }^{139}$

Kritiker der Absprachen führen seit eh und je Verstöße gegen Verfassungs- und Strafrechtsprinzipien an. ${ }^{140} 2005$ mahnte der BGH schließlich eine gesetzliche Regelung an ${ }^{141}$ und Anfang 2009 brachte der Gesetzgeber einen Entwurf in das Gesetzgebungsverfahren ein, der z. T. erhebliche Kritik erfuhr. ${ }^{142}$ Trotzdem trat am 4.8.2009 das Gesetz zur Regelung der Verständigung im Strafverfahren in Kraft.

134 Gerlach, Absprachen im Strafverfahren, Ein Beitrag zu den Rechtsfolgen fehlgeschlagener Absprachen im Strafverfahren, 1992, S. 17/18; Kremer (Fn. 58), S. 1; Schünemann (Fn. 46), S. 1895.

135 So z. B. Fezer, Inquisitionsprozess ohne Ende? Zur Struktur des neuen Verständigungsgesetzes, NStZ 2010, S. 177.

136 Viering, Absprachen als verfahrensökonomische Lösung des Schuldnachweisproblems im Strafverfahren. Voraussetzungen und Grenzen unter Berücksichtigung der ökonomischen Analyse des Rechts, 2009, S. 2; Niemöller/Schlothauer/Wieder, Gesetz zur Verständigung im Strafverfahren. Kommentar, 2010, Einl., S. 5; bereits 1982 erschien ein unter dem Pseudonym „Detlef Deal aus Mauschelhausen“ veröffentlichter Beitrag, StV 1982, S. 545 f.

137 Fezer (Fn. 135), S. 178; Hildebrandt (Fn. 53), S. 43.

138 Weigend (Fn. 124), S. 774; Kintzi (Fn. 86), S. 309: „Konjunktur“ der Absprachen.

139 Weigend, Eine Prozessordnung für abgesprochene Urteile?, NStZ 1999, S. 63.

140 Einen Überblick darüber bieten Landau/Eschelbach, Absprachen zur strafrechtlichen Hauptverhandlung, NJW 1999, S. 324/325; Schünemann, Wetterzeichen einer untergehenden Strafprozesskultur? Wider die falsche Prophetie des Absprachenelysiums, StV 1993, S. 658; Müller (Fn. 1), S. 85-169; Hassemer, Pacta sunt servanda - auch im Strafprozess? - BGH NJW 1989, 2270, JuS 1989, S. 892; Eser, Funktionswandel strafrechtlicher Prozessmaximen: Auf dem Weg zur „Reprivatisierung“ des Strafverfahrens?, ZStW 104 (1992), S. 373; Steinhögl, Der strafprozessuale Deal. Perspektiven einer Konsensorientierung im Strafrecht, S. 16 ff.; Schünemann (Fn. 109), B 80 ff.; Weigend (Fn. 138), S. 776 ff.

141 BGHSt 50, 40 = JZ 2005, S. 628; Meyer-Goßner (Fn. 46), Einl., Rn. 119 g.

142 Burhoff, Regelung der Verständigung im Strafverfahren, ZAP 2009, S. 477; Fischer, Absprache-Regelung: Problemlösung oder Problem?, StraFo 2009, S. $177 \mathrm{ff}$. 


\section{Kritische Würdigung des neuen Gesetzes (insbesondere des $§ 257$ c StPO)}

Durch das Verständigungsgesetz wurde die Absprachenpraxis als Alternative zum streitigen Verfahren geschaffen (in Prantls Worten: „... aus dem Strafgesetzbuch ein Handelsgesetzbuch" 143 gemacht.). Die neue Regelung ist von einer großen Widersprüchlichkeit geprägt. So soll der Schuldspruch nicht Gegenstand einer Verständigung sein ( 257 c Abs. 2 S. 3 StPO); gleichzeitig wird jedoch nicht ausgeschlossen, dass die Staatsanwaltschaft im Rahmen ihrer gesetzlichen Befugnisse auch von $\S 154$ StPO Gebrauch machen könne. ${ }^{144} \S 257$ c Abs. 1 S. 1 StPO ermöglicht, dass sich das Gericht mit den Verfahrensbeteiligten über das Ergebnis des Verfahrens verständigen kann. Andererseits soll der Amtsermittlungsgrundsatz des $\S 244$ Abs. 2 StPO unberührt bleiben, $\S 257$ c Abs. 1 S. 2 StPO. ${ }^{145}$ Dass sich das nicht verträgt, ist offensichtlich. ${ }^{146}$

Problematisch ist weiterhin, dass sich die Verfahrensbeteiligten entgegen der bisherigen Rechtsprechung des BGH gemäß $§ 257$ c Abs. 1 S. 1 StPO nun auch dann verständigen können, wenn kein Geständnis des Angeklagten vorliegt (§257 c Abs. 1 S. 1 StPO ist lediglich eine Soll-Vorschrift). ${ }^{147}$ Soweit ein - immerhin - vorliegendes Geständnis nicht durch eine Beweisaufnahme auf seine Richtigkeit hin überprüft wird (und das ist sehr selten!), wird zudem das ursprüngliche Ziel des Strafverfahrens, die materielle Wahrheit zu finden, aufgegeben. Die Möglichkeit der Legitimation kann dann nur noch in der Verständigung selbst zu suchen sein. ${ }^{148}$ Äußerst bedenklich stimmt schließlich auch der Umstand, dass das später über die Schuld- und Straffrage entscheidende Gericht selbst Partner der Abspracheverhandlung ist. Der Richter verliert so als Verhandlungspartner seine neutrale Stellung und ist nach einer gescheiterten Absprache voreingenommen, sodass das Prinzip der Unabhängigkeit des Richters augenscheinlich verletzt ist. ${ }^{149}$ Zudem verletzt das Erfordernis der staatsanwaltlichen Zustimmung den Art. 92 GG und den Gewaltenteilungsgrundsatz aus Art. 20 Abs. 2 S. 2 2. Hs. GG. ${ }^{150}$ Nach Prantl wird der Gerichtssaal zum Markt, auf dem die Strafen ausgehandelt werden. Neben dem Strafrecht gälten künftig nun die auf dem Markt herrschenden Prinzipien: Do ut des - „Ich gebe Ihnen ein Geständnis, Sie geben mir dafür eine milde Strafe“. So habe jeder etwas vom „Deal“. Nur die Gerechtigkeit stehe dumm da, aber sie sei ja sowieso nicht Verfahrensbeteiligte. ${ }^{151}$

143 Prantl (Fn. 1), S. 4.

144 BT-Drucks. 16/12310, S. 13; Strate (Fn. 1), S. 365, spricht von „Verlogenheit“.

145 Wohlers, Das Strafverfahren in den Zeiten der „Eilkrankheit“, NJW 2010, S. 2474; Leipold, Die gesetzliche Regelung der Verständigung im Strafverfahren, NJW-Spezial 2010, S. 521; Kudlich (Fn. 42), C 60.

146 So z. B. Fezer (Fn. 135), S.183; ähnlich Strate (Fn. 1), S. 365.

147 Leipold (Fn. 145), S. 520; Bittmann, Das Gesetz zur Regelung der Verständigung im Strafverfahren, wistra 28 (2009), S. 415.

148 Fezer (Fn. 135), S. 179/180.

149 Schünemann, Ein deutsches Requiem auf den Strafprozess des liberalen Rechtsstaats, ZRP 2009, S. 107; ähnlich auch schon Weßlau (Fn. 123), S. 167.

150 So auch Leipold (Fn. 145), S. 521; Prantl (Fn. 1), S. 4 geht sogar so weit, zu behaupten, dass die den Richtern verliehene Unabhängigkeit kein Bequemlichkeitsgrundrecht sei.

151 Prantl (Fn. 1), S. 4; ähnlich auch Hamm, Absprachen im Strafverfahren, ZRP 1990, S. 342, der von „kaufmännischen Tugenden“ auf der Richterbank spricht. 
Abschließend kann und muss festgestellt werden, dass die von den Kritikern seit eh und je vorgebrachten Bedenken gegen Absprachen nicht beseitigt werden konnten. Der Gesetzgeber hat sich lediglich darauf beschränkt, die Vorgaben, die der BGH entwickelt hat, in Gesetzesform zu fassen. ${ }^{152}$ Es ist daher sehr zweifelhaft, ob sich die Absprachenpraxis durch die neuen Normen beschränken lassen wird. ${ }^{153}$ Anstatt informelle Absprachen als rechtswidrig zu verbieten, hat man der Verständigung nun den offiziellen Weg in die Strafprozessordnung geebnet. Somit wurde diese ,aus der materialen Rechtsstaatlichkeit verabschiedet". ${ }^{154}$

\section{Legitimation von Absprachen?}

Gibt es nun einen Weg, wie sich Absprachen legitimieren lassen? Das Prinzip der materiellen Wahrheit und das Schuldprinzip kommen augenscheinlich als Legitimationsgrundlagen nicht in Betracht. Bei einer Absprache wird gerade auf die weitere Sachaufklärung verzichtet, ohne dass das Gericht zu einer vollen Überzeugung über den Geschehensablauf gelangt ist. ${ }^{155}$ Es steht die auf Konsens und Kooperation angelegte Suche nach irgendeiner Wahrheit im Vordergrund. ${ }^{156}$

Die neue Regelung der Verständigung könnte jedoch durch das Konsensprinzip legitimiert werden, sodass der Vorwurf der Verfassungswidrigkeit noch aus dem Weg geräumt werden könnte. Einige Autoren nehmen das an. Die Konsensmaxime wird hiernach als weiterer Verfahrensgrundsatz des deutschen Strafprozesses anerkannt. ${ }^{157}$ Andere sehen wegen des Verweises auf die Amtsaufklärungspflicht des $\S 244$ Abs. 2 StPO ein ,wesentliches Bollwerk“ gegen ein Konsensprinzip. ${ }^{158}$ Zudem gelte das Konsensprinzip auch deswegen bei einer Absprache nicht, da der Angeklagte der Verurtei-

152 Wohlers (Fn. 145), S. 2474; Fezer (Fn. 135), S. 178; Fischer, Ein Jahr Absprache-Regelung. Praktische Erfahrungen und gesetzlicher Ergänzungsbedarf, ZRP 2010, S. 251; Schünemann (Fn. 149), S. 105; Altenhain/Haimerl, Die gesetzliche Regelung der Verständigung im Strafverfahren - eine verweigerte Reform, JZ 2010, S. 327.

153 Wohlers (Fn. 145), S. 2474; in diese Richtung auch schon Fischer, Regelung der Urteilsabsprache - ein Appell zum Innehalten, NStZ 2007, S. 435.

154 Albrecht (Fn. 77), S. 156; Albrecht (Fn. 7), S. 265; a. A. Jahn/Müller, Das Gesetz zur Regelung der Verständigung im Strafverfahren - Legitimation und Reglementierung der Absprachenpraxis, NJW 2009, S. 2631 sehen das Gesetz als großen Schritt in die richtige Richtung.

155 Fornauf(Fn. 2), S. 211.

156 Fornauf(Fn. 2), S. 209.

157 Jahn/Müller (Fn. 154), S. 2625/2631; Jahn (Fn. 128), S. 287, der Absprachen als Sonderfall des allgemeinen praktischen Diskurses (nach Habermas) bezeichnet; auch BRAK-Entwurf, S. 3; abrufbar: www.brak.de/seiten/pdf/Stellungnahmen/2006/Stn25.pdf (abgerufen am 9.9.2011), vgl. auch BRAK, ZRP 2005, S. 235 ff.

158 Kudlich, Erfordert das Beschleunigungsgebot eine Umgestaltung des Strafverfahrens? Verständigung im Strafverfahren - Fristsetzung für Beweisanträge - Beschränkung der Geltendmachung von Verfahrensgarantien, NJW-Beil. 2010, S. 88. 
lung nicht aus innerer Überzeugung zustimme, sondern nur, um einer schwereren Strafe zu entgehen. ${ }^{159}$

Im Gegensatz zu den oben angesprochenen konsensualen Verfahren beziehen sich Absprachen überwiegend auf komplexe Fälle vor dem Landgericht, sind also den oben genannten Verfahren mangels Bagatellcharakters gar nicht zugänglich. Bei § 153 a StPO und dem Strafbefehlsverfahren muss der Beschuldigte zudem der angebotenen Rechtsfolge zustimmen, damit Rechtskraft entsteht. Absprachen werden dagegen mit einem autoritativen richterlichen Ausspruch beendet, der unabhängig vom Beschuldigten durchgesetzt werden kann. ${ }^{160}$ Somit scheint es in diesem Kontext verfehlt, vom Konsensprinzip zu sprechen, sodass dieses auch nicht die Anwendung der Rechtsfolge legitimieren kann ${ }^{161}$ - mag auch eine gewisse Konsensorientierung den Absprachen aufgrund der Mitwirkung des Beschuldigten ( $\$ 257$ c Abs. 3 S. 4 StPO) nicht ganz fremd sein. Es liegt jedoch keine echte Selbstunterwerfung des Beschuldigten vor. ${ }^{162}$ Zusammenfassend: Weder das Prinzip der materiellen Wahrheit noch ein Konsensgedanke können die Rechtsfolge einer Verständigung rechtfertigen: Beides steht in striktem Gegensatz zu einem an der Verfassung orientierten rechtsstaatlichen Strafrecht.

\section{Konflikt des Konsens- und des Opportunitätsprinzips mit tragenden Verfassungs- und Strafrechtsprinzipien}

Im Folgenden wird aufgezeigt, mit welchen Verfassungs- und Strafrechtsprinzipien die Opportunitätsvorschriften und die konsensualen Vorschriften in Konflikt geraten.

\section{Schuldprinzip}

Mit dem Schuldprinzip soll sichergestellt werden, dass Strafe Schuld, d. h. individuelle Vorwerfbarkeit, voraussetzt. Es stellt das Maßprinzip eines rechtsstaatlichen Strafrechts dar, um so den staatlichen Zugriff auf den Einzelnen zu legitimieren. ${ }^{163}$ Bei den hier dargestellten konsensualen Verfahren $\S \S 153 \mathrm{a}, 407 \mathrm{ff}$. StPO beruht die Legitimation staatlicher Sanktionen wie gesehen nicht mehr auf nachgewiesener individueller Schuld, ${ }^{164}$ da auf der Grundlage eines hinreichenden Tatverdachts abgeurteilt wird, sondern - wenn überhaupt - auf einem Konsens zwischen den Beteiligten. Beide Verfahren verletzen somit das Schuldprinzip.

159 Meyer-Goßner (Fn. 46), Einl., Rn. 119 h, § 257 c, Rn. 3, der von einem bloßen „Lippenbekenntnis" spricht; ähnlich auch Fezer (Fn. 135), S. 179; Schünemann (Fn. 149), S. 106; Weßlau, Konsensprinzip als Leitverfahren des Strafverfahrens, StraFO 2007, S. 4; a. A. Niemöller/Schlothauer/Wieder/Niemöller (Fn. 136) § 257 c, Rn. 72.

160 Fornauf(Fn. 2), S. 209; so auch Weßlau (Fn. 88), S. 34; Deiters (Fn. 21), S. 18.

161 So auch Fornauf (Fn. 2), S. 209, ders.(Fn. 57), S. 227: bei Absprachen handele es sich um einen konsentierten Abbruch der Wahrheitsfindung; Weßlau (Fn. 88), S. 34.

162 Fornauf(Fn. 2), S. 209.

163 Fornauf (Fn. 2), S. 55; Fornauf (Fn. 57), S. 219.

164 Fornauf (Fn. 57), S. 225; ähnlich bezüglich des Strafbefehlsverfahrens Geis (Fn. 107), S. 193. 
Das ist bei $\S 153$ StPO anders, weil im Zusammenhang mit der Verfahrenseinstellung keinerlei Sanktionierung erfolgt. ${ }^{165}$ Somit kann $\S 153$ StPO das Schuldprinzip nicht verletzen.

\section{Gewaltenteilung, Art. 20 Abs. 2 GG und Rechtsprechungsmonopol/ Richtervorbehalt, Art. 92 GG}

Die Idee der Verteilung der Staatsmacht (und damit der gegenseitigen Kontrolle) auf die drei Gewalten Legislative, Exekutive und Judikative stammt aus der Zeit der Aufklärung und geht vor allem auf Montesquieu ${ }^{166}$ zurück. ${ }^{167}$ Heute besteht die Gewaltenteilung in der Verteilung der Ausübung der Staatsgewalt und in der Zuweisung der jeweiligen Funktionen auf verschiedene Staatsorgane. ${ }^{168}$ Das Rechtsprechungsmonopol des Art. 92 GG konkretisiert das Gewaltenteilungsprinzip und steht den Richtern zu. Die Aufgabenbereiche von Richtern und Staatsanwälten sind somit zu trennen. Dies entspricht dem ursprünglichen Gedanken, das Inquisitionsverfahren durch den Anklageprozess zu ersetzen. ${ }^{169}$ Staatliche Eingriffe in die persönliche Rechtssphäre von Betroffenen unterliegen zudem grundsätzlich einem Richtervorbehalt, denn erst die richterliche Überprüfbarkeit und Kontrolle und deren Billigung kann staatliche Eingriffe legitimieren. ${ }^{170}$

$\S 153$ a StPO verleiht dem Staatsanwalt schon im Ermittlungsverfahren die Befugnis, Rechtsfolgen zu verhängen, die bisher nur vom Richter auferlegt werden durften. Insofern ist eine Schwächung des Richtervorbehalts zu verzeichnen. ${ }^{171}$ Fraglich ist, ob der Staatsanwalt damit „Strafgerichtsbarkeit“ ausübt. Wäre dies der Fall, läge eine Verletzung des Art. 92 GG und des Art. 20 Abs. 2 S. 2 2. Hs. GG vor. ${ }^{172}$ Das überwunden geglaubte Inquisitionsprinzip hätte gleichsam durch die Hintertür wieder Einzug gehalten. ${ }^{173}$ Sieht man $\S 153$ a StPO jedoch richtigerweise nicht als Opportunitäts-, sondern als Konsensvorschrift an, ${ }^{174}$ ist eine Verletzung der Art. 92 GG und Art. 20 Abs. 2 S. 2 2. Hs. GG nicht ausgeschlossen. Auch aus der Notwendigkeit der gerichtlichen Zustimmung zur Einstellung nach $\S 153$ a StPO kann sich nichts anderes ergeben, denn Art. 92 GG verlangt, dass eine ,rechtsprechende“ Entscheidung allein und in allen

165 Auch bei dem Verfahren nach $\S 153$ StPO muss die Tat nicht vollständig aufgeklärt sein; es muss lediglich eine Schuldhypothese bestehen. Vgl. auch Ioakimidis (Fn. 91), S. 33.

166 Montesquieu, Vom Geist der Gesetze (De l'ésprit des lois), aus dem Französischen übersetzt von A. W. Hauswald, 1829 (Nachdruck von 1748).

167 Pott (Fn. 19), S. 12; Fornauf (Fn. 2), S. 66-72.

168 Pott (Fn. 19), S. 12.

169 Männlein (Fn. 37), S. 150/151.

170 Fornauf (Fn. 2), S. 101; Kolz, Einwilligung und Richtervorbehalt, 2006, S. 125; vgl. vertiefend dazu auch: Talaska, Der Richtervorbehalt: Ein sinnvolles Element des Grundrechtsschutzes?, 2007, S. $54 \mathrm{ff}$.

171 Kausch (Fn. 28), S. 42 ff.; Männlein (Fn. 37), S. 148; Bohnert (Fn. 21), S. 231; Fornauf (Fn. 57), S. 223.

172 Kausch (Fn. 28), S. 43/44; Männlein (Fn. 37), S. 148; Sinner (Fn. 33), S. 273: „Richter vor dem Richter".

173 Männlein (Fn. 37), S. 151; LR/Beulke (Fn. 38), § 153 a, Rn. 12.

174 S. vorher z. B. Kausch (Fn. 28), S. 62. 
Punkten vom Richter getroffen wird. ${ }^{175}$ Bei $\S 153$ a StPO hingegen ist der Richter nicht an der primären Entscheidungsfindung beteiligt, sondern kann lediglich seine Zustimmung zu deren Ergebnis erteilen oder unterlassen. $\mathrm{Zu}$ den Garantien des Art. 92 GG gehört gerade auch, dass der einer rechtsprechenden Entscheidung zugrundeliegende Sachverhalt durch ein unabhängiges Gericht festgestellt wird. ${ }^{176}$ Die Einstellung nach $\S 153$ a StPO bewirkt daher eine grundlegende Funktionsverschiebung zwischen Richter und Staatsanwalt. Dem Richter kommen von vornherein nur noch relativ schwerwiegende Delikte zu und die Entscheidung darüber, was als schwerwiegend zu beurteilen ist, liegt jetzt nicht mehr bei ihm, sondern beim Staatsanwalt. ${ }^{177}$ Zudem entsteht auch eine Verschiebung der relevanten Entscheidungen aus der Hauptverhandlung in das nicht-öffentliche Ermittlungsverfahren, ${ }^{178}$ sodass die traditionelle Form der Hauptverhandlung immer mehr zur statistischen Ausnahme wird. Dies ist insofern bedenklich, als gerade dieses mit zahlreichen rechtsstaatlichen Garantien ausgestattet ist. ${ }^{179}$ Sowohl eine Verletzung des Gewaltenteilungsprinzips als auch eine Verletzung des Art. 92 GG liegen somit vor. ${ }^{180}$

Auch durch andere konsensuale Verfahren, wie die Absprachen (§ 257 c StGB) oder das Strafbefehlsverfahren, wird die kontradiktorische Struktur des Strafverfahrens ausgehebelt. Eine gegenseitige Kontrolle von Exekutive und Judikative findet nicht mehr statt, sodass auch diese Verfahrenstypen in ihrer derzeitigen Ausgestaltung das Gewaltenteilungsprinzip verletzen. ${ }^{181}$ Zudem zieht die Staatsanwaltschaft mit Wahl des Strafbefehlsverfahrens die materielle Entscheidungsbefugnis an sich. Das Fehlen einer Hauptverhandlung führt weiterhin zu hohen Einbußen der dem Richter zur Verfügung stehenden Entscheidungsgrundlagen, da er in der Hauptsache nur auf die Ermittlungsakten der Staatsanwaltschaft zurückgreifen kann. ${ }^{182}$

175 Kausch (Fn. 28), S. 67; Männlein (Fn. 37), S. 150; BVerfGE 22, 49, 73 ff.

176 Kausch (Fn. 28), S. 68/70; ähnlich Fezer (Fn. 45), S. 26; SK/Weßlau (Fn. 7), vor $\S \S 151$ ff, Rn. 16.

177 Kausch (Fn. 28), S. 134; Erb (Fn. 23), S. 104; Albrecht, Die Kriminalisierung der Dritten Gewalt. Ein verfehlter Beitrag der Exekutive zur Steigerung der Funktionstüchtigkeit des Kriminaljustizsystems, ZRP 2004, S. 259; Titz, Weisungsfreie Staatsanwälte, KritV 2010, S. 261.

178 Weigend (Fn. 27), S. 115; so auch Fornauf (Fn. 2), S. 101 und Hassemer (Fn. 27), S. 531.

179 Satzger, Braucht der Strafprozess Reform?, StraFO 2006, S. 46; Satzger, Chancen und Risiken einer Reform des strafrechtlichen Ermittlungsverfahrens. Gutachten C für den 65. Deutschen Juristentag, 2004, C 17.

180 Auf den ersten Blick scheint auch $\S 153$ StPO dem Staatsanwalt Kompetenzen zuzuweisen, die denen des $\S 153$ a StPO ähneln, sodass ihm eine „quasi-richterliche“ Rolle zukommt. Jedoch sind die durch $\S 153$ StPO verliehenen Befugnisse im Vergleich zu denen des $\S 153$ a StPO eher gering, da keine eigenständigen Sanktionen gesetzt werden können. Bedenklich erscheint jedoch, dass die Aussonderung des nichtstrafwürdigen Unrechts im Ermessen des Staatsanwalts steht. Die Straflosigkeit solcher Fälle müsste obligatorisch sein. Vgl. hierzu Kausch (Fn. 28), S. 223/224; Albrecht, Exekutivisches Recht. Eine Einführung in empirische Analysen zur staatsanwaltlichen Diversion in Nordrhein-Westfalen, in: ders. (Hrsg.), Informalisierung des Rechts - Empirische Untersuchungen zur Handhabung und zu den Grenzen der Opportunität im Jugendstrafrecht, 1990, S. 48.

181 So auch Fornauf(Fn. 57), S. 225; jedoch bleibt im Gegensatz zu § 153 a StPO beim Strafbefehl die Entscheidung formal dem Richter vorbehalten, s. Fornauf (Fn. 2), S. 171.

$182 \operatorname{Müller}$ (Fn. 101), S. 56; Ebert (Fn. 117), S. 206; Taubald (Fn. 4), S. 45. 
Bei den konsensualen Verfahren wird die rechtsprechende Gewalt den Richtern faktisch entzogen, obwohl Art. 92 GG sie „den Richtern anvertraut“. Der Einfluss anderer Verfahrensbeteiligter fließt durch Konsensualisierung unbestimmbar in die rechtsprechende Gewalt ein, sodass das Urteil nicht mehr durch den Richter, sondern durch das Prinzip des Konsenses und seiner rechtsstaatswidrigen Zwänge hergestellt wird. Art. 92 GG und Art. 20 Abs. 2 S. 2 2. Hs. GG sind somit verletzt.

\section{Unschuldsvermutung, Art. 6 Abs. 2 EMRK und Rechtsstaatsprinzip}

Die Unschuldsvermutung nach Art. 6 Abs. 2 EMRK verlangt, dass niemand als schuldig behandelt werden darf, solange nicht seine Schuld durch ein rechtskräftiges Urteil eines ordentlichen Gerichts nachgewiesen ist. ${ }^{183}$ Eine Verletzung der Unschuldsvermutung durch $\S 153$ StPO ist nicht ganz fernliegend, da die Klärung des gegen den Beschuldigten bestehenden Verdachts nicht so weit wie möglich durchgeführt wird, ${ }^{184}$ seine mögliche Unschuld also gar nicht bewiesen werden kann. Der Betroffene hat einen Anspruch, von jeglichem Verdacht gemäß $§ 170$ Abs. 2 StPO freigesprochen zu werden, wenn es nicht zu einer Verurteilung kommt. Darin und in der dauerhaften Datenerfassung des Beschuldigten liegt eine Verletzung der Unschuldsvermutung und des Rechtsstaatsprinzips. $^{185}$

$\S 153$ a StPO setzt nicht voraus, dass die Schuld feststeht, sondern es genügt ein hinreichender Tatverdacht. Die Stimmen, die den Strafcharakter von Weisungen und Auflagen verneinen, kommen hier wiederum zu dem Ergebnis, dass kein Verstoß gegen die Unschuldsvermutung vorliegt, auch, da die Zustimmung des Beschuldigten die Legitimationsgrundlage bilde. ${ }^{186}$ Die Zustimmung des Beschuldigten entsteht jedoch nicht unbedingt freiwillig und kann somit nicht als Legitimationsgrundlage dienen. ${ }^{187}$ Sieht man Auflagen und Weisungen richtigerweise als strafähnliche Eingriffe an, so wird mangels sicheren Nachweises der Schuld bei $\S 153$ a StPO Art. 6 Abs. 2 EMRK verletzt, da erstere auch potentiell Unschuldige treffen. ${ }^{188}$

Auch für den Erlass eines Strafbefehls ist nach richtiger Auffassung ${ }^{189}$ lediglich ein hinreichender Tatverdacht erforderlich. Der Strafbefehl, gegen den kein Einspruch erhoben wird, steht einem rechtskräftigen Urteil gleich (§ 410 Abs. 3 StPO). Der Strafbefehl geht also von der Schuld des Betroffenen aus, ohne dass dessen Schuld in einem umfassenden Verfahren festgestellt worden wäre. Der hinreichende Tatverdacht kann diese Kriminalsanktion mangels Tatnachweises nicht legitimieren. Eine Verletzung des Art. 6 Abs. 2 EMRK ist also auch hier gegeben. ${ }^{190}$

183 Männlein (Fn. 37), S. 158; Rose (Fn. 21), S. 76; Pott(Fn. 19), S. 143; BVerfGE 22, 254, 265.

184 A. A. Kühl, Unschuldsvermutung, Freispruch und Einstellung, 1983, S. 89.

185 Horstmann (Fn. 17), S. 153.

$186 \mathrm{Kunz}$, Das strafrechtliche Bagatellprinzip. Eine strafrechtsdogmatische und kriminalpolitische Untersuchung, 1984, S. 74/75.

187 Männlein (Fn. 37), S. 160.

188 Rose (Fn. 21), S. 77; Dencker (Fn. 59), S. 150; so dann auch Fezer (Fn. 45), S. 26, der jedoch den Strafcharakter der Auflagen und Weisungen ablehnt.

189 S. dazu vorher.

190 Ebert (Fn. 117), S. 259-263; so auch Ambos (Fn. 106), S. 288. 


\section{Richterliche Unabhängigkeit, Art. $97 G G(\S 1$ GVG, $\S 25$ DRiG)}

Art. 97 GG richtet sich heute gegen alle Versuche der Staatsgewalten, auf die richterliche Entscheidung Einfluss zu nehmen ${ }^{191}$ und stellt eine Teilkonkretisierung des Gewaltenteilungsgrundsatzes dar. Eine Begrenzung der richterlichen Unabhängigkeit ist nur im freiheitlichen Gesetz zu sehen. ${ }^{192}$ Die richterliche Unabhängigkeit wurde ursprünglich im Kampf gegen die absolutistische Kabinettsjustiz zur Abwehr monarchistischer Übergriffe in die Rechtssprechung erstritten. ${ }^{193}$

Die Folgen der beschriebenen Entwicklungen und der Anwendung der $\S \S 153$, 153 a, 257 c, 407 ff. StPO liegen im Verlust einer effektiven Kontrolle von staatlicher und gesellschaftlicher Macht. ${ }^{194}$ Aus der beschriebenen Verlagerung von der Hauptverhandlung in das staatsanwaltliche Ermittlungsverfahren, welches auf Flexibilität, Schnelligkeit und Effizienz angelegt ist, folgt ein umfassender Bedeutungsverlust der Unabhängigkeit der Dritten Gewalt, da die ausführliche Rechtskontrolle staatlicher Ermittlungsmaßnahmen durch den Richter nicht mehr stattfindet. ${ }^{195}$

Es lässt sich ein pessimistisches Fazit ziehen: Die Eingriffe in die Struktur des deutschen Strafprozesses durch Opportunitäts- und Konsensprinzip beeinflussen das Richterbild entscheidend. Der Richter wird neben dem Verteidiger ,,konsensualer Manager des scheinbar Vernünftigen“. ${ }^{196}$ Auch nach Fornauf besteht die richterliche Entscheidung nur noch in einem formalen - weil gesetzlich noch vorgesehenen - Bestätigen des exekutivischen Sanktionierungsvorschlags. Dem Richter komme allenfalls noch die Rolle eines Urkundsbeamten zu, der inhaltlich keinerlei Einfluss auf den Ausgang des Verfahrens nehmen könne. So werde der unabhängige Richter ,zum Büttel der Exekutive“. ${ }^{197}$ Nach Salditt hätte der konsensuale Prozess - und das gilt in gleicher Weise für das Opportunitätsprinzip - dann richtigerweise dort, wo sie zur Regel würden, das Richteramt und damit zugleich das Verfahren zerstört. ${ }^{198}$

\section{Prinzip der materiellen Wahrheit und Amtsermittlungsgrundsatz}

Das Ziel des Strafverfahrens wird herkömmlich in der Durchsetzung des materiellen Rechts gesehen. Damit ist das Finden einer materiell richtigen Entscheidung gemeint, die prozessordnungsgemäß zustande kommt und damit letztlich der Schaffung von Rechtsfrieden dienen soll. Die Entscheidung muss sich dabei am Maßstab der Gerech-

191 Dreier, Grundgesetz. Kommentar, Band III, Art. 83-146, 2000 - Dreier/Schultze-Fielitz, Art. 97, Rn. 15.

192 Albrecht (Fn. 27), S. 149/150; Dreier/Schultze-Fielitz (Fn. 191), Art. 97, Rn. 14; Thomas, Richterrecht, 1986, S. 7; Papier, Die richterliche Unabhängigkeit und ihre Schranken, NJW 2001, S. 1089.

193 Papier (Fn. 192), S. 1089.

194 Albrecht (Fn. 27), S. 152.

195 Fornauf(Fn. 57), S. 231; ähnlich auch Albrecht, Erosionen des rechtsstaatlichen Strafrechts, KritV 2010, S. 43.

196 Albrecht (Fn. 7), S. 266; auch Albrecht (Fn. 5), S. 46: „gesprächsleitende Ausgleichsperson".

197 Fornauf(Fn. 57), S. 226.

198 Salditt (Fn. 5), S. 75. 
tigkeit orientieren. ${ }^{199}$ Nach dem Prinzip der materiellen Wahrheit, das einfachgesetzlich in $\S 244$ Abs. 2 StPO normiert ist, muss das Erforschen der Wahrheit die notwendige Grundlage für ein gerechtes Urteil sein. Nur die wahrheitsgemäße Ermittlung des jeweiligen Tatgeschehens kann die Grundlage für eine an der Strafgerechtigkeit ausgerichtete Rechtsfolge sein. ${ }^{200}$

Der Grundsatz der materiellen Wahrheit ist bei den konsensualen Verfahren der $\S 153$ a und $\S \S 407 \mathrm{ff}$. StPO insoweit betroffen, als dass auf eine umfassende Wahrheitsermittlung durch eine in richterlicher Unabhängigkeit geleitete Hauptverhandlung einschließlich einer Beweisaufnahme verzichtet wird und die Schuldfrage letztlich offen bleibt. Vielmehr wird auf der Grundlage einer formellen, u. U. zwischen den Verfahrensbeteiligten ausgehandelten Wahrheit eine Sanktion verhängt. ${ }^{201}$ Die gleichen Bedenken kommen auch bezüglich der Opportunitätsvorschrift $\S 153$ StPO auf. Auch hier wird die Wahrheitsermittlung vorzeitig abgebrochen, sodass die materielle Wahrheit nie zu Tage treten wird.

\section{Bestimmtheitsgebot, Art. 103 Abs. 2 GG, § 1 StGB und Gleichheitsgrundsatz, Art. 3 Abs. 1 GG}

Bedenken ergeben sich bei den $\S \S 153,153$ a StPO weiterhin daraus, dass der Gesetzgeber die Ermessensentscheidungen von Voraussetzungen abhängig gemacht hat, die nur in unbestimmten Rechtsbegriffen (z. B. ,geringe Schuld“, „Schwere der Schuld“, „öffentliches Interesse“) genannt sind. Somit ist der Anwendungsbereich unbestimmt, sodass die Gefahr einer ungleichen Anwendung seitens der Staatsanwaltschaft besteht. ${ }^{202}$ In einigen Fällen wird die Verletzung einer Norm geahndet, in anderen nicht. Zudem sind erhebliche regionale Unterschiede zu verzeichnen. ${ }^{203}$ Auch führen konsensuale und opportune Erledigungsformen in der Regel unvermeidbar zu einer ZweiKlassen-Justiz mit erheblichen Vorteilen für begüterte Beschuldigte. ${ }^{204}$ So können finanziell besser gestellte Täter z. B. Auflagen leichter erfüllen. Eine Verletzung des Bestimmheitsgebots und des Gleichheitsgrundsatzes sind somit gegeben.

\section{E. Rechtfertigung durch andere Legitimationen?}

Da die Verletzungen der Verfassungs- und Strafrechtsprinzipien durch die beschriebenen Verfahren evident sind und deren Rechtsfolgen auch nicht durch das Schuldprinzip

199 Fornauf(Fn. 2), S. 60.

200 Fornauf (Fn. 2), S. 61; Fornauf (Fn. 57), S. 220.

201 Fornauf(Fn. 2), S. 205; Weßlau (Fn. 88), S. 46.

202 Fezer (Fn. 45), S. 25; ähnlich auch schon Kausch (Fn. 28), S. 152; Sinner (Fn. 33), S. 110; SK/Weßlau (Fn. 7), § 153, Rn. 8; Albrecht (Fn. 7), S. 213; Hildebrandt (Fn. 53), S. 134/135; Shin (Fn. 17), S. 28.

203 Böttcher (Fn. 12), S. 299, Fn. 4.

204 Gössel, Über die Pflicht der materiell-objektiven Wahrheit und die Zuständigkeiten zur Eröffnung eines Strafverfahrens und zu dessen Durchführung, in: Eser/Goydke/Maatz/ Meurer (Hrsg.), Strafverfahrensrecht in Theorie und Praxis. Festschrift für Lutz MeyerGoßner zum 65. Geburtstag, 2001, S. 194; besonders deutlich wird dies bei § 153 a StPO. 
und das Prinzip der materiellen Wahrheit legitimiert werden können, ist zu fragen, ob es alternative Legitimationsmöglichkeiten gibt.

\section{Legitimation durch das Opportunitätsprinzip?}

An dieser Stelle ist noch einmal zu hinterfragen, ob das Opportunitätsprinzip in seiner momentanen Ausgestaltung als Legitimationsprinzip für vorzeitige folgenlose (!) Einstellungen in Frage kommt. In dem rechtsstaatlichen Modell eines klassischen Vergeltungsstrafrechts und dem diesem zugrundeliegenden Prinzip der Strafgesetzlichkeit können vorzeitige Verfahrenseinstellungen aus Opportunitätsgründen, die gegen Verfassungs- und Strafrechtsprinzipien verstoßen, nicht gerechtfertigt werden. Jedoch ist zu bedenken, dass das Strafrecht angesichts der massiven Ausweitung materieller Überlastung zu „kollabieren“ droht. ${ }^{205}$ Eine Möglichkeit, diesen Konflikt zu lösen, wäre, ein rechtsstaatlich verträgliches Opportunitätsprinzip einzuführen. Wichtig wäre in diesem Kontext vor allem, für hinreichende Bestimmbarkeit zu sorgen, der Staatsanwaltschaft weniger Ermessen einzuräumen, um Gleichheit in der Einstellungspraxis zu garantieren, und den Anwendungsbereich auf Fälle außerhalb des Kernstrafrechts zu begrenzen. Zudem müsste die Anwendung einer solchen Opportunität auf den Verzicht der weiteren Strafverfolgung begrenzt sein und gerade nicht die Verhängung von Sanktionen und damit weitergehende Zwecksetzungen erlauben. ${ }^{206} \mathrm{Ob}$ diese Vorgehensweise in der Praxis realisierbar ist, bleibt zu bezweifeln. In der momentanen Ausgestaltung taugt das Opportunitätsprinzip jedoch nicht als Legitimationsgrundlage für eine Einstellung nach $\S 153$ StPO. Die rechtsstaatsgefährdenden Nebenfolgen, die vor allem im Vorabschnitt herausgearbeitet wurden, sind zu gravierend.

\section{Konsensprinzip als Legitimationsgrundlage für den Schuldspruch?}

Schließlich kommt das Konsensprinzip als Legitimationsgrundlage für die Rechtsfolgen der hier dargestellten konsensualen Vorschriften in Betracht. Diese bedürfen insofern einer Legitimation, als die gegenüber dem Beschuldigten verhängten Rechtsfolgen eine staatliche - ihn belastende - Maßnahme darstellen. Schuldprinzip und Prinzip der materiellen Wahrheit sollen nun durch die alternative Legitimationssuche nach einem Konsens mit dem Beschuldigten ersetzt werden. ${ }^{207}$

Fraglich ist jedoch, ob das Konsensprinzip überhaupt als Legitimationsgrundlage geeignet ist. Das Konsensprinzip zielt darauf, das Defizit der verfahrensrechtlichen Schuldüberzeugung eines unabhängigen Richters auszugleichen. In beiden dargestellten Verfahren wird nämlich nur eine Verdachtssanktion verhängt. ${ }^{208} \mathrm{Ob}$ die Rechtsfolge

205 Fornauf(Fn. 2), S. 178/179; ähnlich auch Sinner (Fn. 33), S. 118.

206 Fornauf (Fn. 2), S. 180/181/183; ähnlich Hassemer (Fn. 27), S. 539.

207 Fornauf (Fn. 57), S. 224/226; Salditt, Der Griff nach dem Vorurteil - Frühe Genugtuung und Schlichtung als strafprozessuales Reformprogramm, StV 2002, S.276; Weßlau (Fn. 88), S. 30: durch $\S 153$ a StPO sei das Prinzip der materiellen Wahrheit bereits durch das Konsensprinzip ersetzt worden.

208 Fornauf(Fn. 2), S. 205. 
eines Strafbefehls über das Konsensprinzip gerechtfertigt werden kann bzw. muss, hängt insbesondere von der Frage ab, ob ein hinreichender Tatverdacht für den Erlass eines Strafbefehls ausreicht oder ob der Richter von der Schuld des Täters überzeugt sein muss. Nach richtiger Ansicht ist ein hinreichender Tatverdacht ausreichend, der fehlende Schuldnachweis soll dann durch die nachträgliche Zustimmung des Beschuldigten kompensiert werden. ${ }^{209}$ Das kontradiktorische Verfahren, an dem in unserem Rechtsstaat festgehalten werden muss, und das Konsensprinzip schließen sich jedoch für die Legitimation staatlicher Sanktionen schlichtweg aus. Das Konsensprinzip darf die Idee des Schuldprinzips in einem Rechtsstaat nicht ersetzen; genau das will es jedoch mit der Legitimierung einer Verdachtsstrafe beim Strafbefehlsverfahren erreichen. Somit kann das Konsensprinzip in einem rechtsstaatlichen Strafrecht nicht die Verhängung einer Sanktion i. S. d. $\S \S 407$ ff. StPO legitimieren. ${ }^{210}$

Wie beim Strafbefehl soll auch bei $\S 153$ a StPO das Konsensprinzip die Legitimation für eine mit der Verfahrenseinstellung verbundene strafähnliche Sanktion liefern. ${ }^{211}$ Höchst bedenklich stimmt jedoch der Umstand, dass aufgrund der Drucksituation, in die der Beschuldigte bei dem „Angebot“ des $§ 153$ a StPO gerät, nicht mehr von einer Freiwilligkeit der Zustimmung gesprochen werden kann. ${ }^{212}$ Ein derartig unechter, durch Drohung mit einer weitaus höheren Strafe, durchgesetzter Konsens reicht für die teils gravierenden Rechtsfolgen des $\S 153$ a StPO nicht aus. ${ }^{213}$ Die Zustimmung des Beschuldigten wird sich in erster Linie darauf beziehen, die Auflagen und Weisungen zu erfüllen und soll gerade kein Eingeständnis seiner Schuld bedeuten. Nur, weil der Beschuldigte die Sanktion erfüllt, bedeutet das also nicht, dass er schuldig ist. ${ }^{214}$ Somit kann auch die Rechtsfolge des $\S 153$ a StPO nicht durch das Konsensprinzip legitimiert werden. Für die Absprachen im späteren Strafprozess (§ 257 c StPO) gelten diese Bedenken in gleicher Weise.

Nach diesen Feststellungen ist nach der Daseinsberechtigung des Konsenses im Strafverfahren bzw. danach zu fragen, ob im Strafprozess überhaupt ein richtiger Konsens existieren kann. Nach Weigend könne man nicht wirklich von einem „Konsens“ sprechen. $\mathrm{Zu}$ beachten sei nämlich, dass der Beschuldigte typischerweise den Strafverfolgungsbehörden institutionell in jeder Hinsicht unterlegen sei. Beide unterschieden sich in unaufhebbarer Weise hinsichtlich ihrer Möglichkeiten, übereinander Macht auszu-

209 Fornauf(Fn. 2), S. 157/158, s. vorher.

210 So auch Fornauf (Fn. 2), S. 162/163; so i. E. dann auch KK/Fischer (Fn. 12), § 408, Rn. 15, da dem Konsenselement durch $\S 411$ Abs. 2 S. 2 i.V.m. $\S 420$ die Legitimation entzogen worden sei; a. A. Weßlau (Fn. 88), S. 200-202, die das gegenwärtige Strafbefehlsverfahren als „Beispiel für eine Integration des Konsensprinzips in ein Verfahren, das am Prinzip der materiellen Wahrheit orientiert ist", sieht.

211 Fezer (Fn. 45), S. 33: die innere Legitimation sei in der freiwilligen Erfüllung der Auflagen zu sehen; ähnlich Saliger (Fn. 45), S. 166/167; Taubald (Fn. 4), S. 32.

212 Salditt (Fn. 207), S. 276: wenn der Konsens durch Druck herbeigeführt werde, bestehe er nur zum Schein; Schünemann, Wohin treibt der deutsche Strafprozess?, ZStW 114 (2002), S. 60: „unechter Konsens“; a.A. Fezer (Fn. 45), S. 33; Eser (Fn. 140), S. 383; Saliger (Fn. 45), S. 167.

213 Schünemann (Fn. 212), S. 60.

214 Fornauf (Fn. 2), S. 197/198; so auch bereits Dencker (Fn. 59), S. 150. 
üben. ${ }^{215}$ Zwischen dem die Strafverfolgung betreibenden Staat und dem Bürger bestehe ein unüberwindliches Machtgefälle. Die sogenannte „einvernehmliche Erledigung“ stelle in Wirklichkeit eine Unterwerfung des Beschuldigten dar. Dieser finde sich bestenfalls aus „Einsicht in die Notwendigkeit“" bereit, einer Sanktion zuzustimmen, die ihm als relativ günstig dargestellt wird. ${ }^{216}$

Das Konsensprinzip stellt objektiv zu gewährleistendes Recht in die Disposition des Beschuldigten. ${ }^{217}$ Eine Disposition über den Verfahrensgegenstand kann und darf es aber im Strafprozess nicht geben, da Gegenstand des Strafverfahrens nicht ein Rechtsstreit ist, sondern ein Lebenssachverhalt, der sich als gravierender Normbruch, also als Unrecht, darstellt. ${ }^{218}$ Zudem wäre es befremdend, wenn man sich unter Berufung auf eine „freiwillige Einwilligung“ des Betroffenen einfach von rechtsstaatlichen Mindeststandards befreien könnte. ${ }^{219}$ Das Konsensprinzip ist demnach weder geeignet, die Rechtsfolge der $\S \S 407 \mathrm{ff}$. StPO oder des $\S 153$ a StPO noch diejenige einer Absprache (§257 c StPO) zu rechtfertigen. Das Konsensprinzip ist somit insgesamt als Legitimationsprinzip in einem Rechtsstaat nicht geeignet. ${ }^{220}$

\section{F. Lösungsansatz materiell-rechtliche Entkriminalisierung}

Sowohl das Opportunitätsprinzip als auch das Konsensprinzip bemühen sich um eine Beschleunigung des Strafverfahrens. Eine Straffung soll dadurch erreicht werden, dass Verfahrensrechte des Beschuldigten beschnitten werden und/oder vom streitigen Verfahren in ein konsensuales Verfahren ausgewichen wird. Grund für diese Entwicklung ist wie gesehen in erster Linie der Umstand, dass die Strafjustiz stark überlastet ist. Anstatt sich aber jedoch (nur) auf die prozessuale Ebene zu konzentrieren, die rechtsstaatliche Grundprinzipien des Strafverfahrens aufweicht, sollte man vielmehr analysieren, was die Ursachen für diese Überlastung sein könnten. So kommen eine Überdehnung des materiellen Strafrechts, ${ }^{221}$ an die das Strafverfahren nicht angepasst wurde, und die ständige Neuzuweisung von Steuerungsansprüchen an das Strafrecht in Betracht. Dieser Entwicklung, so wird zunehmend richtigerweise gefordert, sei durch eine materiell-rechtliche Entkriminalisierung zu begegnen. ${ }^{222}$

215 Weigend (Fn. 3), S. 304; ähnlich Salditt (Fn. 5), S. 75: dem durch Macht hergestellten Konsens fehle die Legitimation; auch ähnlich Sinner (Fn. 33), S. 268 f.

216 Weigend, Reform des Strafverfahrens, ZStW 104 (1992), S. 500; Weßlau (Fn. 88), S. 136; ähnlich Salditt (Fn. 5), S. 75, der konstatiert, dass dem durch Macht hergestellten Konsens die Legitimation fehle und das scheinbare Einvernehmen in sich brüchig sei.

217 Fornauf(Fn. 2), S. 201 und auch noch einmal nachdrücklich S. 281.

218 Mit Weßlau (Fn. 159), S. 5.

219 So auch Duttge (Fn. 59), S. 560.

220 A. A. Weßlau (Fn. 159), S. 4, die das Konsensprinzip als "zweite Legitimationsstufe", dort, wo es um Begrenzungen des Beweisstoffes geht, ansieht.

221 Wohlers (Fn. 145), S. 2470; so auch Kudlich (Fn. 158), S. 87 und Kudlich (Fn. 42), C 21, der die Zunahme von Straftatbeständen im Kernstrafrecht und die Einführung komplexer Strafvorschriften im Nebenstrafrecht wie bspw. $\S 20$ a, 38, 39 WpHG beklagt.

222 Albrecht (Fn. 195), S. 180/181; Naucke, Das System der prozessualen Entkriminalisierung, in: Samson/Dencker/Frisch/Frister/Reiß (Hrsg.), Festschrift für Gerald Grünwald zum siebzigsten Geburtstag, 1999, S. 413; so i. E. auch Kudlich (Fn. 158), S. 87; Salditt (Fn. 5), S. 66. 
So hat Ambos auf materiell-rechtlicher Ebene dreierlei Verfahrensverkürzungen vorgeschlagen: Die Beseitigung bestimmter Tatbestände (vollständige Entkriminalisierung), die Herabstufung bestimmter Verhaltensweisen zu bloßem Verwaltungsunrecht (Ordnungswidrigkeiten) und die Unterwerfung von bestimmten Verhaltensweisen unter andere, nicht kriminalstrafrechtliche, Sozialkontrollen (partielle oder beschränkte Entkriminalisierung). ${ }^{223}$ Auch die Hessische und die Niedersächsische Kommission haben bereits vor fast 20 Jahren vorgeschlagen, dass Effizienzsteigerung nicht durch Opportunität, sondern durch Zurücknahme übersteigerter und überkommener Regelungsansprüche (Entkriminalisierung) erreicht werden soll. So werden bspw. die Einführung eines materiell-rechtlichen Geringfügigkeitsprinzips, die Einschränkung der Strafbarkeit fahrlässigen Verhaltens und normative Entkriminalisierungen vorgeschlagen. ${ }^{224}$

Albrecht sieht die richtige Diagnose aus dem „Dilemma von ökonomischer Überlast, kriminalpolitisch-dogmatischer Unzulänglichkeit, normativer Überkommenheit und den Allmachtsanspruch präventiver Übersteuerung gesellschaftlicher Probleme“" in einem „Heckenschnitt überwuchernder Unzulänglichkeiten“, womit ebenfalls eine normative Entkriminalisierung gemeint ist. ${ }^{225}$ Ziel sollte die Reduzierung auf das Kernstrafrecht und die Entlastung des Strafverfahrens durch Zurücknahme übersteigerter und überkommener Regelungsansprüche sein. Verfahrensrechtliche Entlastungen sollten nur insoweit stattfinden, als sie rechtsstaatliche Grundprinzipien des Strafverfahrens unberührt ließen. Zudem müssten entliberalisierende Gesetze zum Straf- und Strafprozessrecht wieder rückgängig gemacht werden. ${ }^{226}$

Auch Weigend sieht die Prävention als „Wiege“ des Problems an: Der moderne Staat greife in viele neue Gebiete lenkend ein und setze das Strafrecht als Steuerungsinstrument ein. Zu diesem Zweck schaffe der Gesetzgeber ständig neue Strafvorschriften, deren Durchsetzung von vornherein gar nicht möglich sei und auch nicht beabsichtigt werde. $^{227}$ In dieselbe Richtung argumentiert auch Naucke treffend: Es gebe im Besonderen Teil des StGB und im Nebenstrafrecht nur noch wenige Vorschriften, die nicht nach dem Prinzip ,Zwar in großem Umfange strafbar, aber unter bestimmten Bedingungen nicht zu bestrafen" gefasst seien. ${ }^{228}$

Worin genau liegen nun die Vorteile einer materiell-rechtlichen Entkriminalisierung gegenüber der vom Gesetzgeber und der Justiz gewählten prozessualen Entkriminali-

223 Ambos (Fn. 106), S. 282; so auch Baumann (Fn. 26), S. 273 f.; ähnlich Hirsch, Zur Behandlung der Bagatellkriminalität in der Bundesrepublik Deutschland. Unter besonderer Berücksichtigung der Stellung der Staatsanwaltschaft, ZStW 92 (1980), S. 245 ff., der u. a. die Neuschaffung einer dritten Deliktskategorie unterhalb des Vergehens („Verfehlung“) fordert.

224 Albrecht (Fn. 195), S. 181; Albrecht (Fn. 77), S. 127 f.; Albrecht/Beckmann/Frommel/Goy/ Grünwald/Hannover/Holtfort/Ostendorf: Strafrecht - ultima ratio. Empfehlungen der Niedersächsischen Kommission zur Reform des Strafrechts und des Strafverfahrensrechts, 1992, S. 15-74; Albrecht/Hassemer/Voß (Fn. 4), S. 8, 9, 10-131; ähnlich Naucke (Fn. 222), S. 413; Roos, Entkriminalisierungstendenzen im Besonderen Teil des Strafrechts, 1981, S. 309.

225 Albrecht (Fn. 5), S. 48.

226 Albrecht (Fn. 77), S. 141/142; auch Hassemer (Fn. 27), S. 538.

227 Weigend (Fn. 138), S. 780.

228 Naucke (Fn. 222), S. 417; genannt werden hier u. a. § 142 Abs. 4, Abs. 6; § 261 Abs. 1, Abs. 9, Abs. 10 StGB; $\S \S 306$ ff StGB. 
sierung? Zunächst ist anzumerken, dass eine Ungleichbehandlung wegen unterschiedlicher Anwendung der $\S \S 153,153 \mathrm{a}, 257 \mathrm{c}, 407 \mathrm{ff}$. StPO in den Ländern vermieden werden könnte, da allein materielle Vorschriften einheitlich für das gesamte Bundesgebiet gelten würden. Weiterhin könnten nur dann sanktionierende Maßnahmen verhängt werden, wenn die Schuld des Täters feststünde, sodass der Verstoß gegen die Unschuldsvermutung und gegen das Schuldprinzip ausgeräumt würde. Die Staatsanwaltschaft könnte auch nicht mehr eigenständig Sanktionen verhängen, sodass das Gewaltenteilungsprinzip und der Richtervorbehalt gewahrt blieben. Die Entscheidung, welches Verhalten als strafbar angesehen wird und welches nicht, würde wieder dem Gesetzgeber zufallen, der hierzu von der Verfassung auch befugt ist, und nicht mehr der Exekutive. Durchgesetzt würde diese Entscheidung dann wieder durch einen unabhängigen Richter. Das wichtigste Anliegen der Opportunitäts- und Konsensvorschriften, die Justizentlastung, würde schließlich dadurch erreicht, dass es zu bestimmten Delikten, die nicht mehr mit Strafe bedroht wären, gar keine Ermittlungen mehr anzustellen gäbe, weder von Seiten der Polizei, noch von der Staatsanwaltschaft noch vom Richter. ${ }^{229}$ Die Opportunitäts- und Konsensvorschriften wären durch eine materiellrechtliche Entkriminalisierung, die nur manifestes Unrecht im Sinne eines Kernstrafrechts strafrechtlich eingefasst lässt, überflüssig und könnten gestrichen werden.

\section{G. Ausblick}

\section{Ablehnung formeller Entkriminalisierung mittels Opportunität und Konsens}

Bereits durch die bestehenden Opportunitäts- und Konsensvorschriften werden elementare Verfassungs- und Strafrechtsprinzipien verletzt. Dies ist der Preis für ein ausschließliches Effizienzdenken der Strafverfolgung. ${ }^{230}$ Zwar gibt es Überlegungen, das Opportunitätsprinzip rechtsstaatlicher zu machen. Jedoch ist sehr fraglich, ob sich Opportunität jemals mit einem Verfahren, das am Prinzip der materiellen Wahrheit ausgerichtet ist, vereinbaren lässt. Die Antwort fällt wohl negativ aus. Ebenfalls unklar ist, ob ein konsensuales Verfahren rechtsstaatlicher gemacht werden kann. Gegen den Vertrags- und den Konsensgedanken im Strafverfahren spricht im Wesentlichen Folgendes: Sobald schützenswerte Interessen der Allgemeinheit im Spiel sind, kann eine Entscheidung nicht mehr dem mitunter geradezu willkürlichen Belieben der Prozessbeteiligten überlassen bleiben. Im Strafprozess geht es um rückwirkende Feststellung zentralen Unrechts im Wege der verfahrensförmigen, rechtsstaatlichen Rekonstruktion des ,wahren" Geschehens. ${ }^{231}$

Die Konsensorientierung und der Trend zur Opportunität gefährden die traditionellen Zielsetzungen des rechtsstaatlichen Strafrechts enorm. Dem „,neuen“ Strafprozess geht es um Opportunität und Effizienz. ${ }^{232}$ Das Strafbefehlsverfahren und das Verfahren nach $\S 153$ a StPO waren ursprünglich nicht auf einen Konsens ausgerichtet. Das Strafbe-

229 Männlein (Fn. 37), S. 183-185.

230 Albrecht, Das Strafrecht im Zugriff populistischer Politik, in: Vom unmöglichen Zustand des Strafrechts, 1995, S. 441.

231 Vgl. im Ergebnis auch Duttge (Fn. 59), S. 552.

232 Sinner (Fn. 33), S. 137. 
fehlsverfahren wurde als ein verkürztes, summarisches Verfahren angesehen, welches gleichwohl eine richterliche Schuldüberzeugung und die materielle Wahrheit im Blick hatte. §153 a StPO war ursprünglich als Opportunitätsvorschrift eingeführt worden. Dass aus beiden Verfahren schließlich konsensuale Verfahren wurden, hat seinen Grund in ihrer stetigen Ausweitung hinsichtlich des Anwendungsbereichs und hinsichtlich der zu verhängenden Rechtsfolgen ${ }^{233}$ - vor dem Hintergrund der präventiven Überlastung des Kriminaljustizsystems. Das Strafrecht verlagert seine Steuerungsansprüche vom materiellen Recht in das Prozessrecht. Die der Effizienzsteigerung geschuldete Informalisierung des Strafverfahrens ${ }^{234}$ hat die Bindung staatlicher Macht an das Recht auf extreme Weise geschwächt. So wird von der „Erosion rechtsstaatlicher Prinzipien“ ${ }^{\text {235 }}$ gesprochen: Tragende Verfassungs- und Strafrechtsprinzipien geraten immer mehr in Vergessenheit, bis sie ganz aufgeweicht sind.

Dem vom Gesetzgeber gewählten Weg einer formellen - im soziologischen Sinne richtiger ,informellen' - Entkriminalisierung ist somit entschieden entgegenzutreten. Die beschriebenen Verfahren sind ernsthaft zu überdenken und idealerweise abzuschaffen. Wenn man sie jedoch - trotz Verfassungswidrigkeit in der momentanen Ausgestaltung - weiterhin praktiziert, sollte man wenigstens darauf achten, sie nicht in Fällen anzuwenden, in denen eine mittlere oder schwere Schuld vorliegt, sondern sie zumindest auf ihren ursprünglich vorgesehenen Anwendungsbereich, die Bagatellkriminalität, zu beschränken.

\section{II. „Königsweg“.236 Materielle Entkriminalisierung und Rückzug des Strafrechts aus einem präventiven Steuerungsanspruch}

Das Problem, nämlich die übermäßige Weite der Strafvorschriften, liegt wie gesehen im materiellen Bereich. Daher sollte man auch eine Lösung im materiellen Strafrecht suchen. Wählt man diesen Weg, geht man systematisch korrekt vor und vermeidet vor allem den Widerspruch, dass einerseits ein Verhalten im materiellen Recht mit Strafe bedroht ist, andererseits jedoch auf seine Nicht-Verfolgung im Verfahren gesetzt wird. ${ }^{237}$ Zudem werden die Verfassungs- und Strafrechtsprinzipien gewahrt. Der „Königsweg“ im Umgang mit Massen- und Bagatellkriminalität bleibt also die Anpassung des materiellen Rechts. ${ }^{238}$ Soll das Strafrecht nicht völlig zerbrechen, bleibt zudem nur sein Rückzug aus dem ,allumfassenden präventiven Steuerungsanspruch“. ${ }^{239}$ Nur dann ist das Strafverfahren nach rechtsstaatlichen Prinzipien zu führen. Dies ist die Strafjustiz den Menschen schuldig.

233 Fornauf (Fn. 2), S. 231; so auch Heinz (Fn. 11), S. 300.

234 Albrecht (Fn. 180).

235 Albrecht (Fn. 195), S. 165.

236 Dieser Begriff geht zurück auf Albrecht (Fn. 180), S. 39.

237 Weigend (Fn. 27), S. 106/107; ähnlich: Lorenzen (Fn. 23), S. 548/549; auch Ambos (Fn. 106), S. 283; s. auch in anderen Rechtsordnungen $\S 46$ ö. StGB und Art. 26 poln. StGB; einen guten Überblick über bereits erfolgte materielle Entkriminalisierung bietet Roos (Fn. 224), S. $58 \mathrm{ff}$.

238 Albrecht (Fn. 180), S. 39; Albrecht/Hassemer/Voß (Fn. 4), S.7; Fornauf (Fn. 2), S. 180; so auch Schünemann (Fn. 212), S. 59.

239 Albrecht (Fn. 195), S. 180; Albrecht/Hassemer/Voß (Fn. 4), S. 103. 